\title{
Transport behavior-mining from smartphones: a review
}

\author{
Valentino Servizi* (iD, Francisco C. Pereira, Marie K. Anderson and Otto A. Nielsen
}

\begin{abstract}
Background: Although people and smartphones have become almost inseparable, especially during travel, smartphones still represent a small fraction of a complex multi-sensor platform enabling the passive collection of users' travel behavior. Smartphone-based travel survey data yields the richest perspective on the study of inter- and intrauser behavioral variations. Yet after over a decade of research and field experimentation on such surveys, and despite a consensus in transportation research as to their potential, smartphone-based travel surveys are seldom used on a large scale.

Purpose: This literature review pinpoints and examines the problems limiting prior research, and exposes drivers to select and rank machine-learning algorithms used for data processing in smartphone-based surveys.

Conclusion: Our findings show the main physical limitations from a device perspective; the methodological framework deployed for the automatic generation of travel-diaries, from the application perspective; and the relationship among user interaction, methods, and data, from the ground truth perspective.
\end{abstract}

Keywords: Smartphone-based travel surveys, Machine learning, User behavior, Transport, Map-matching, Mode detection, Activity inference, Data fusion

\section{Introduction}

To support the planning, design, and policy-making processes for improving transport systems [44], travel surveys capture essential aspects of user behaviors on which behavioral modeling relies [18]. For designing the representativeness of a user sample under study, the statistical approach in traditional travel surveys is prominent. The process involves person-to-person (P2P) interactions for data collection, a process overlapping with ground truth collection: Trained travel surveyors directly validate data with users and manually reconstruct users' travel-diaries for behavioral study.

In contrast, machine-learning plays a primary role in smartphone-based travel surveys (SBTS). The data collection process involves device-to-device interaction, with machine-learning algorithms automatically

*Correspondence: valse@dtu.dk

Department of Technology, Management and Economics, Technical

University of Denmark (DTU), Kgs. Lyngby, Copenhagen, Denmark reconstructing users' travel-diaries directly from data that might contain various sources of errors [50]. By submitting each travel-diary to the user for validation (i.e., to find out whether the user needs to change the travel-diary or not), the process can collect ground truth through a person-to-device (P2D) interaction between the user and an input/output interface, either via a website or smartphone [59].

Since the introduction of the first generation of smartphones equipped with assisted global positioning systems (AGPS) in the early 2000s, researchers have described smartphone-based travel surveys as a promising platform to measure user transport behavior. They can track the same user with an extended time horizon [91], collect data passively [125], detect previously unreported short trips, and avoid stereotypes of daily activity [104] (e.g., "I don't remember what I did, but here's what I usually do"). Given that SBTS would likely facilitate the discovery of inter- and intra-user behavior variations, the question 
is why SBTS have not yet replaced traditional travel surveys [41].

For researchers and public authorities, standardized performance indexes based on standard datasets support optimal investment decision-making. This approach also applies to classification or regression methods underpinning the identification of user transport behavior variations. Nevertheless, standardization in this field is lacking. Instead, decision-making often relies on assumptions, such as (i) consistent performance indexes evaluation across studies; (ii) comparable performance indexes across studies, even when based on different datasets; (iii) adequate representativeness of the few public datasets available; (iv) exact ground truth. By definition, each necessary assumption represents a knowledge gap.

We ask and answer the following questions: What are the main machine-learning methods that are used in the field? What is the relationship between ground truth and machine-learning methods? What are the primary datasets studied? What characteristics do these datasets have, and what features can we extract from them, and how? What are the challenges for machine-learning in the field of SBTS? What are the main implications for transport science?

To tackle these questions, we proceed by snowballing first forward and then backward [114]. We cover deterministic and machine-learning methods based on different datasets collected from across the world. We examine how models and algorithms exploit various data sources such as AGPS, inertial navigation systems (INS), geographic information systems (GIS), and Internet-of-Things.

The paper analyzes technologies enabling SBTS data validation, such as data preparation and feature extraction, and focuses on machine-learning methods for mining user's behavior from smartphone data. These methods target why people travel, where along the transport network they travel, and which mode of transport they use. These technologies make an impact by reducing resources associated with running traditional travel surveys, while enhancing users' transport behavior dataresolution. Following this approach, we are able to review purpose imputation, map-matching, and mode detection methods.

Existing literature and reviews offer a clear picture of how algorithms and background technologies evolve to provide improved measures of users' travel behavior variations. For example, we list several specialized methods with impressive performance scores. We also find unilateral perspectives offering standardization pathways for both methods application and performance evaluation. In practice, limitations such as data representativeness, ground truth quality, and performance evaluation procedures may often result in a biased perception of each method's potential.

Decisions based on wrong assumptions and biased perceptions represent a threat to the progress of this field. To bridge the gap, we provide the following contributions. We deliver a self-contained overview connecting the user transport behavior measures with the supporting smartphone-sensing-platform. We detail how available methods can be combined to extract behavioral information from various data streams. We show the convergence between research areas studying complementary aspects of transport behavior. We organize each reviewed work by task complexity, method requirements, and dataset representativeness. So we facilitate methods' assessment and comparison across specific use cases, mitigating the limitations of dry and incomparable performance scores. The paper reveals opportunities offered by device-todevice interactions for data validation instead of other interactions, and exposes gaps in deep learning strategic applications.

The first section below presents the dimensions describing transport behavior and the tools embodied in a smartphone device for data collection. The following section describes the methods used to identify transport behavior from data and an overview of the implications for transport science. The subsequent discussion presents a joint look on the results of the surveyed literature, which the conclusion summarizes from a big-data perspective. We include the Tables organizing the main features of the literature reviewed.

\section{Measures and tools}

To support the reader through the following analysis and discussion, we start by providing context and presenting concepts on which the paper rests, i.e., definitions, employment, and technological framework of SBTS.

\subsection{Measures of transport behavior}

The following terms are used to describe a user's journey (throughout a single day, for example; see Fig. 1) and represent the different variables, or measures, that SBTS is used to collect for studies on transport behavior.

\subsubsection{Tour}

Aggregation of trips, such that users' travels start and end at the same place, e.g. at home [28].

\subsubsection{Trip}

Travel entity identified with a set of attributes such as: start-location, start-time, purpose, transport mode, arrival time, arrival location [28]. 


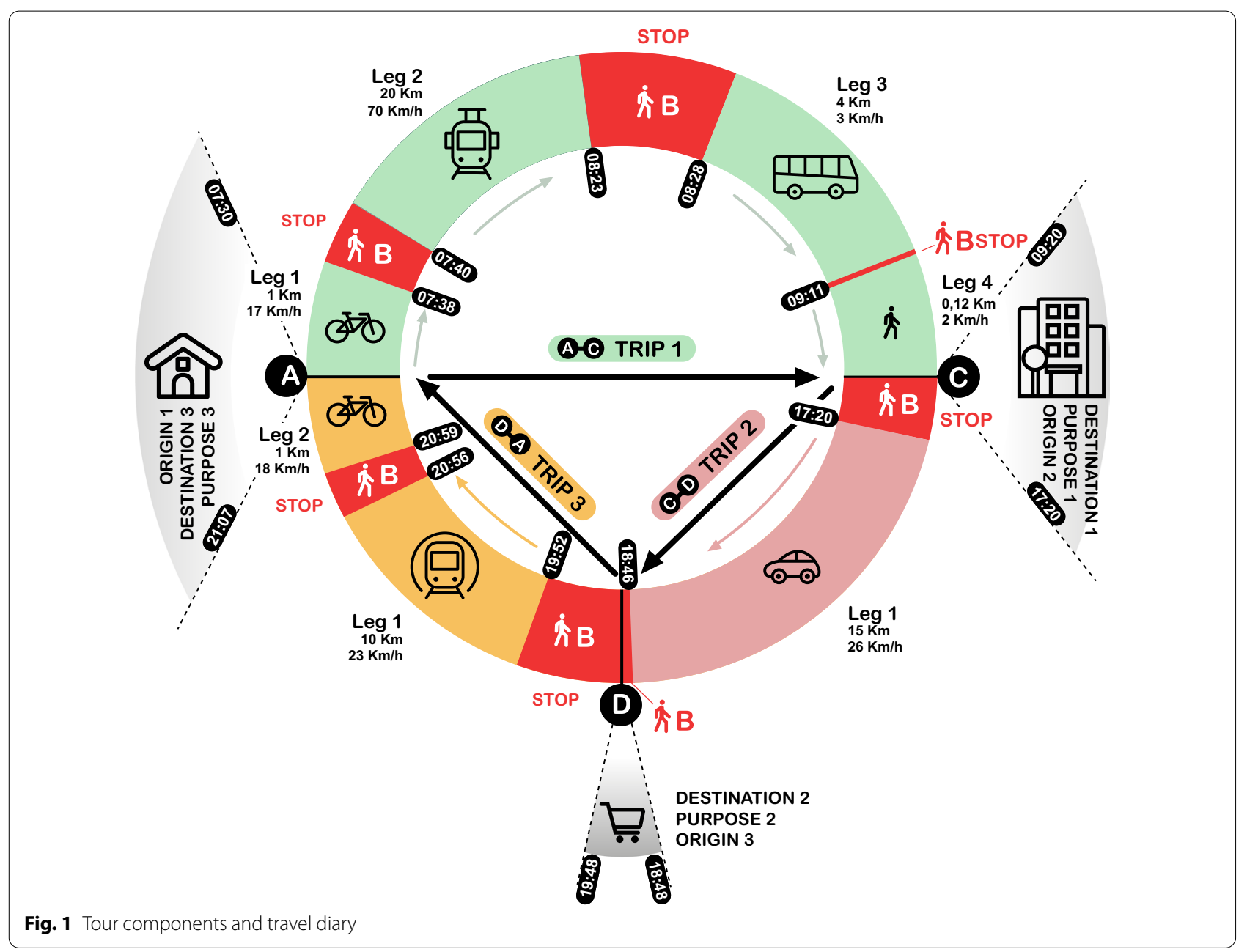

\subsubsection{Leg}

Also identified as a "trip segment," this is the unimodal segment between two stops. Each trip segment has a start-time and -location, end-time and -location, and stop-purpose at the end of the leg (see Fig. 1B) [28, 96].

\subsubsection{Purpose}

This represents what triggers the trip from origin to destination (see Fig. 1A, C, D), and identifies the "activity" performed at the end of a trip.

\subsubsection{Stop}

This can be reduced to two categories: stops at the end of legs (see Fig. 1B), and stops at the end of trips (see Fig. 1A, C, D).

\subsubsection{Transport mode}

This refers to a trip leg [120] and identifies, e.g., walking, cycling, car, train, bus, light rail (see Fig. 1).

\subsubsection{Mode-chain-type}

The literature provides no strict consensus on the definition of this term, and we define it as the list of transport modes one uses to get from the origin to the destination of a trip (see Fig. 1).

\subsubsection{Travel-diary}

This can focus on "one-day" (see Fig. 1) or on "multipledays" and it describes the user trips through: (i) legs, where each leg has a unique transport mode; (ii) purpose; (iii) stops; and (iv) mode-chain-type. Generally, it is linked to a user, and his or her link-able personal information, such as: (i) age; (ii) occupation; (iii) education level; (iv) home address; and (v) work address. [28] presents a detailed list of further personal attributes.

\subsubsection{Ground truth}

This describes the true measurements of the target variables, for example the purpose of a trip, its 
transport-mode-chain, and the route between origin and destination. In general, the literature refers to (i) travel-diary; (ii) prompted recall survey; (iii) user input in mobile phones [12]; (iv) experiments (e.g. mode known) [98]; (v) trips reported in-situ by the user participating in an experiment [33]; and (vi) "traffic counts" extracted from video recordings [53]. However, because ground truth is lacking in several studies [26], authors have introduced alternative methods to close this gap, the results of which serve as a benchmark [54]. In case of synthetic data, studies on map-matching refer to the random selection among a set of alternative shortest paths [71]; in case of real data, other studies refer to GPS receivers collecting two independent measures, where ground truth is the measure with a higher sampling rate [51]. When algorithms target public transportation, ground truth can be extracted as the combination of bus stops and intersections within the transport network [42]. In the best-case scenario, the information is reported by users. As ground truth always seems prone to errors, Prelipcean et al. [87] have introduced the concept of "acceptable truth," which, while not truly absolute, may be considered sufficiently accurate relative to the application.

\subsection{Pioneering smartphone-based travel surveys}

Within the last 20 years, traditional travel survey methods have been subject to the pressure of disruptive technological evolution. The large penetration of smartphone devices equipped with low-cost sensors, the introduction of Web 2.0, and the emergence of other directly related phenomena, such as Big Data [5], could represent a tipping point for this research method [125]. There are several reasons to complement and/or substitute traditional travel surveys with smartphone-based technology, given the former's shortcomings, as follows:

1. Statistic representativeness, improvable or decreasing in some population's strata [80];

2. Trend of unreported short trips which the user tends to forget or does not want to mention [104];

3. Undetected behavior variations of the same user, due to the design of traditional travel surveys, which collects a cross-section sample of the population by focusing on one single day for each respondent [91];

4. Data collection cost per surveyed user [101].

The first large-scale SBTS deployments were the Future Mobility Sensing (FMS) in 2012, and the Sydney Travel and Health Survey in 2013. Most of the SBTS we know offer either web or app validation (seldom both), use machine learning, and are fully automated, as for example: (i) FMS/Mobile Market Monitor [126]; (ii)
TRAVELVU/Trivector [38]; (iii) RMOVE/RSG [23]; (iv) Itinerum [83, 84]; (v) MEILI [87]; (vi) Sydney Travel and Health Survey [45]; (vii) Dutch Mobile Mobility Panel [104]; and (viii) MTL Traject [123].

These SBTS no longer collect ground truth via personto-person interaction. Instead, their interfaces provide users with options to validate travel-diaries accurately generated, and to correct errors of the inaccurate ones, collecting ground truth via person-to-device explicit interaction. Nonetheless, users seem unable to report inaccurate diaries that are too difficult for them to correct on their own [102]. Consequently, the risk of encountering incorrect data within ground truth seems unavoidable for survey data. Regardless of whether available ground truth is acceptable or inaccurate, it is important to assess each application on an individual basis in the context of field research.

Success depends also on users' willingness to keep such an application installed on their smartphones. The main drivers determining the decision of a user to keep applications on his or her device are: (i) The information conveyed through the App; (ii) ease of use; (iii) perceived usefulness; (iv) perceived risks; and (v) general satisfaction of the user experience [10].

In (v) we mention a broad and very relevant field of research in which there is consensus about the negative impact of smartphone battery consumption on the user experience, which affects applications' penetration and drop-out rates. Because of the impact on quality of data collection, we observe the same consensus on battery concerns in the field of SBTS [80]. Also, the need of high resolution data in SBTS clashes with the need for battery efficiency enforced by smartphone platform providers [7].

Due to the highly-accurate trajectories generated by smartphones (e.g., through AGPS) and used by SBTS researchers, users are concerned by the potential for privacy violation. These trajectories often expose very personal information of each surveyed user, thereby presenting new challenges [35] in terms of reconciling a need for high-resolution data and a need to ensure privacy for researchers and users, respectively [88, 95].

\subsection{Smartphone capabilities}

In Fig. 2 we present the abstraction of an SBTS platform. The main platform's components are client and server. The client (see Fig. 2A) enables human interaction, e.g., for user travel diary validation (see Fig. 2A.1), and orchestrates sensors, user-generated data (e.g., location), and computer intelligence models. Processing data locally, the client prevents loss of information, and maximizes privacy (see Fig. 2A.3). A battery efficiency layer tunes and optimizes, e.g. data sampling or network 


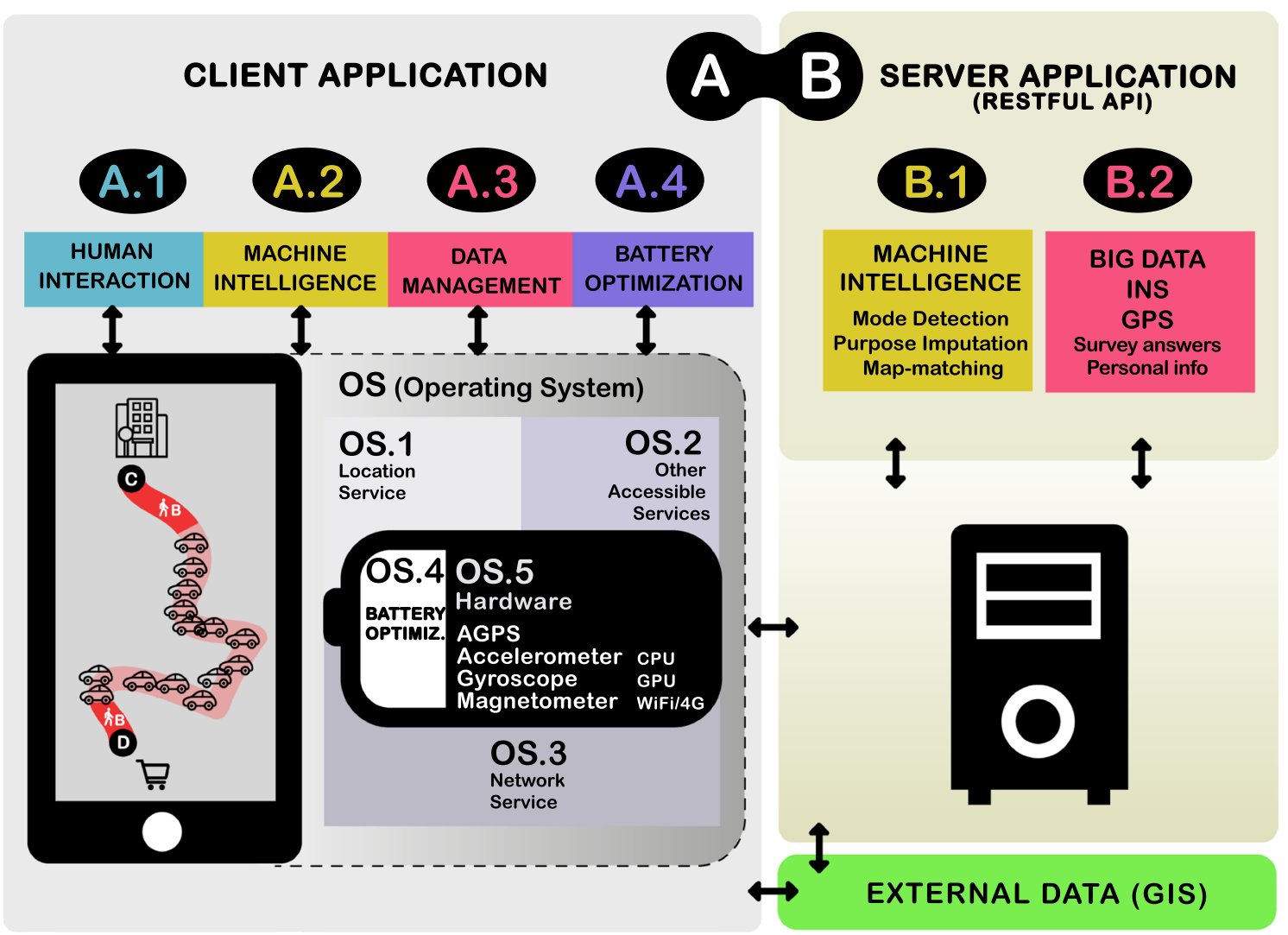

Fig. 2 Smartphone-based travel survey platform architecture

input/output operations among client, server, and external data sources (e.g., GIS).

The sensory system of the platform is the smartphone, represented by:

- Principal hardware components (see Fig. 2OS.5);

- Services exposed by the Operation System (OS, see Fig. 2OS.1-OS.3); and

- Operations beyond users and developers influence, such as those focusing on device battery life extension (see Fig. 2OS.4).

The following list of components is ranked by highest battery consumption to lowest $[85,112]$ :

1. Graphical processing unit (GPU) and screen, triggered when users interact actively with SBTS (e.g., validating travel-diaries).

2. Central processing unit (CPU), engaged also by computer intelligence models for online mode classification, for example, and for detecting conditions to switch off unnecessary sensors. While computation offloading to a server is possible, it implies transmitting data at its own energy cost.
3. AGPS. While GPS depends exclusively on satellites, in smartphones AGPS uses internet to look up the position of satellites and mitigate the cold-start problem. AGPS also uses cell-tower data. This feature is convenient when GPS signal is weak or disturbed, but it introduces challenges for position accuracy. To provide the location of a smartphone while reducing AGPS up-time, several effective strategies are available [82]. Finding the best trade-off between location accuracy, data resolution, and energy consumption is not trivial. Interestingly, we observe a convergence between approaches developed for the OS to improve the energetic efficiency of smartphones, and for datamining to fill data gaps resulting from missing or highly uncertain GPS observations. Both provide location coordinates, reducing GPS sensor need, and leveraging data from INS, GIS, and telecom networks. Nevertheless, some of the current smartphone operation systems do not allow direct access to telecom network data from independent applications [6].

4. Network. An efficient tuning should consider network selection (Cellular or WiFi), data transfer frequency, battery status, and size of the data-transfer. 
5. Accelerometer, gyroscope, and magnetometer raw data is accessible on the main OS platforms. GPS uptime is often optimized by leveraging these sensors to detect whether a user starts or ends a trip [82]. In general, accelerometer and gyroscope readings from smartphones should be collected with a resolution compatible with the motion frequency of human bodies in daily routines, which is above $20 \mathrm{~Hz}$ [49]. The consumption of such high-frequency data streams within the device is not critical for the battery. However, in case of transfer for storage and data consumption offline, handling the number of sensors and the high frequency quickly become critical for the smartphone's battery and for the user's data plan.

Sensors up-time and data transfer to the back-end, as well as the Ground Truth collection on screen are very critical for smartphones battery life [69]. For example, given a fixed data sampling rate, AGPS battery consumption is relatively more sensitive to the up-time, while high frequency sensors consumption is relatively more sensitive to data transfer. If not properly handled within the SBTS, battery drain could occur twice as fast, limiting the battery life to few hours instead of the whole day. Consequently, the impact of service interruptions would result in increasing limitations on the data. Covering the entire day for certain users would no longer be possible, and such a negative user experience would even increase risk of drop-out [10].

\subsection{Physical limitations for data validation}

In addition to the aforementioned battery consumption issues, further critical implications of moving to this new technology are presented below.

\subsubsection{Person-to-device validation}

Design simplicity and intuitiveness should reduce any potential to distract the user while interacting with the survey application, as distractions could impact the quality of ground truth collected [80]. Furthermore, when the purpose of the interaction is directed to amend inaccurate travel-diaries, the impact that the design has on the quality of the ground truth collected from the respondents is even greater. A poor interaction between users and an SBTS interface could trigger a critical loop in which users validate wrong predictions instead of correcting them $[3,30]$.

\subsubsection{Device-to-device validation}

Arising from the convergence of Bluetooth and WiFi protocol in the Internet of Things context, and unlike the classic Bluetooth protocol, Bluetooth low-energy beacons communication is one-to-many (as traditional television or radio), involves few bits of data to be broadcast frequently, and needs no pairing operations. These properties are suitable for proximity detection and interaction with smartphones, and for activity sensing [34, 56]. A pioneering device-to-device ground truth collection on bus trips [66] already experimented Bluetooth lowenergy interaction with SBTS, as an independent and redundant measurement of users' bus trips. This system has the potential to release users' resources that could the be exploited, for example, for filling in context-specific active surveys, and not for validating a travel diary. However, the authors highlight the challenge of finding a signal strength that allows for smartphones to detect beacons in conditions where signals may be attenuated or interfered with. A user's body or location, for example, may attenuate a signal, while interference with other beacons in range could result from passing by a bus stop or grouping with other buses.

\section{Measuring transport behavior}

The primary objective of SBTS consists of accurate ground truth collection from surveyed users. The correct reconstruction of travel-diaries, which encompasses both the transport mode and the purpose of any trip, allows for this goal to be achieved. Research on transport behavior also studies trajectories generated by the same sensors mentioned earlier. Therefore, it applies the same methods described in the following sections. In contrast with SBTS, however, research on transport behavior has the main objective of analyzing behavior, and not of collecting trip ground truth. This subtle difference may support the large community of researchers claiming that mode detection methods should be agnostic to personal and location context (see Tables 1, 2, 3). For example, the same method could generally serve different mode choice studies across the globe. In SBTS, this constraint does not seem to hold since travel-diaries also require predicting each trip's purpose, relying on both sensors and geospatial information (see Table 6). Successful hybrid approaches in this field further expose the shortcomings of such a purist approach. Data preparation is propaedeutic for learning the mode, purpose, and route of any trip. Simultaneously, cross-field convergence proves to be effective; for example, mode detection improves map-matching [26] and purpose imputation tasks [76, 120]. Inversely, map-matching GPS trajectories upfront improves the mode detection task [90]. When outputting a travel diary that allows ground truth collection on users' journeys, we do not find advantages from self-imposing restrictions on what data we should use or what method we should combine. Therefore, we find it beneficial to review purpose imputation and map-matching methods 
Table 1 Classification task ranked by difficulty and score, for mode detection

\begin{tabular}{|c|c|c|c|c|c|c|}
\hline References & No. & Classes & Score & Metric & Validation & Area \\
\hline Zhou et al. [131] & 6 & $\begin{array}{l}\text { Walk, Bike, Bus, Car, Rail, } \\
\text { Plain }\end{array}$ & $86.5 \%$ & Accuracy & Hold-out & Beijing \\
\hline Bohte and Maat [21] & 6 & $\begin{array}{l}\text { Car, Train, Bus-Tram- } \\
\text { Metro, Foot, Bicycle, } \\
\text { Other }\end{array}$ & $70.00 \%$ & Accuracy & n.p. & Netherlands \\
\hline Martin et al. [75] & 5 & Walk, Bike, Bus, Car, Rail & $96.8 \%$ & Accuracy & Manifold-cross-validation & Minnesota \\
\hline Jahangiri and Rakha [55] & 5 & Walk, Bike, Bus, Car, Run & $95.1 \%$ & F-Score & $\begin{array}{l}\text { Manifold-cross-validation, } \\
\text { Out-of-bag-estimate }\end{array}$ & Tennessee \\
\hline Semanjski et al. [96] & 5 & Walk, Bike, Bus, Car, Rail & $94.00 \%$ & Accuracy & Manifold-cross-validation & Leuven \\
\hline Zhou et al. [132] & 5 & $\begin{array}{l}\text { Walk, Bike, Run, in- } \\
\text { Vehicle, Stationary }\end{array}$ & $93.8 \%$ & Accuracy & Hold-out & Georgia (USA) \\
\hline Zhu et al. [134] & 5 & Walk, Bike, Bus, Car, Rail & $93.45 \%$ & F1-Score & Manifold-cross-validation & Beijing \\
\hline Xiao et al. [119] & 5 & $\begin{array}{l}\text { Walk, Bike, el-Bike, Car, } \\
\text { Bus }\end{array}$ & $92.74 \%$ & Accuracy & Manifold-cross-validation & Shanghai \\
\hline Rasmussen et al. [90] & 5 & Walk, Bike, Car, Bus, Rail & $92.4 \%$ & Accuracy & n.p. & Copenhagen \\
\hline Yazdizadeh et al. [122] & 5 & $\begin{array}{l}\text { Walk, Bike, Public transit, } \\
\text { Car, Car and Public transit }\end{array}$ & $88.00 \%$ & $\begin{array}{l}\text { F1-Score weighted } \\
\text { average }\end{array}$ & Manifold-cross-validation & Montreal \\
\hline Dabiri and Heaslip [31] & 5 & Walk, Bike, Bus, Car, Rail & $84.8 \%$ & F-Score & Manifold-cross-validation & Beijing \\
\hline Byon and Liang [22] & 5 & $\begin{array}{l}\text { Auto, Bus, Streetcar, Bike, } \\
\text { Walk }\end{array}$ & $82.00 \%$ & $\begin{array}{l}\text { F1-Score weighted } \\
\text { average }\end{array}$ & Hold-out & Toronto \\
\hline Thomas et al. [104] & 5 & Walk, Bike, Bus, Car, Rail & $82.00 \%$ & Accuracy & n.p. & Netherlands, [43] \\
\hline Dabiri et al. [32] & 5 & $\begin{array}{l}\text { Walk, Bike, Bus, Drive, } \\
\text { Train }\end{array}$ & $76.4 \%$ & $\begin{array}{l}\text { F1-Score weighted } \\
\text { average }\end{array}$ & Manifold-cross-validation & Beijing \\
\hline Jiang et al. [57] & 4 & Walk, Bike, Bus, Car & $98.00 \%$ & Accuracy & Hold-out & Beijing \\
\hline Assemi et al. [11] & 4 & Walk, Bike, Bus, Car & $94.7 \%$ & Accuracy & Hold-out & New-Zealand \\
\hline Yazdizadeh et al. [123] & 4 & Walk, Bike, Transit, Car & $91.8 \%$ & Accuracy & Manifold-cross-validation & Montreal \\
\hline Mäenpääa et al. [72] & 4 & Walk, Bike, Bus, Car & $90.7 \%$ & F1-Score & $\begin{array}{l}\text { Manifold-cross-validation, } \\
\text { Out-of-bag-estimate }\end{array}$ & $\begin{array}{l}\text { Beijing. } 1 \text { week BUS tra- } \\
\text { jectories, } 1000 \text { trajectories } \\
\text { from Open Street Map } \\
\text { (OSM) }\end{array}$ \\
\hline Yazdizadeh et al. [124] & 4 & Walk, Bike, Transit, Car & $83.4 \%$ & Accuracy & Manifold-cross-validation & Montreal \\
\hline
\end{tabular}

in this context. Tables 4, 5 and 6 present purpose imputation; Tables 7, 8 and 9 map-matching methods.

\subsection{Smartphone data mining}

Due to the disparity of progress drivers, we see a trend of increasing fragmentation, inconsistencies, availability, and volume of travel data. In response to this challenge, two main branches seem to arise as flip sides of the same coin $[39,58,64,98]$. The first focuses towards data fusion, intended to compose and then mine high dimensional datasets collected from multiple sources, including GIS, INS, and GPS. The second targets the development of, for example, very sophisticated computer intelligence models, feature extraction methodologies, and optimal hyper parameters selection. These are constantly improving and therefore complementing traditional statistical methodologies, often substituting them for specific purposes [59].
Literature has shown that smartphone data is affected by several errors. For example, map-matching observations based on positions generated by a Nokia N95 would be much less reliable than those based on a dedicated GPS logger [19]. With current smartphones, however, the situation has improved substantially. For mode detection, neural network classifiers have shown higher performance on data collected from smartphones than from GPS devices [22]. Nevertheless, we should be aware that raw sensor measurements may vary between smartphones, as well as within the same model of smartphone [20]. Any measurement is affected by noise that is not necessarily random, since it may be correlated with: weather conditions; building density, materials, and height; crowdedness; physical placement of the smartphone (e.g. in the pocket is different than on a table); smartphone model; and software "bugs." Therefore, achieving consistency of machine-learning methods across different smartphones requires a rigorous 
Table 2 Dataset ranked by number of users, for mode detection

\begin{tabular}{|c|c|c|c|c|c|c|c|}
\hline References & Person-day & Users & Ground truth & Observations & Time & Area & Smartphone App \\
\hline Semanjski et al. [96] & 24,900 & 8303 & $\begin{array}{l}\text { Validated-by- } \\
\text { respondents }\end{array}$ & $\begin{array}{l}\text { 30,000 trips } \\
3,960,243 \text { GPS } \\
\text { points } 340,000 \mathrm{~km}\end{array}$ & n.p. & Leuven & Routecoach \\
\hline $\begin{array}{l}\text { Yazdizadeh et al. } \\
{[124]}\end{array}$ & 88,630 & 6846 & $\begin{array}{l}\text { Validated-by- } \\
\text { respondents } \\
\text { (102,904 trips) }\end{array}$ & 623,718 trips & $\begin{array}{l}2 \text { months collec- } \\
\text { tion period }\end{array}$ & Montreal & MTL Traject App \\
\hline $\begin{array}{l}\text { Yazdizadeh et al. } \\
\text { [123] }\end{array}$ & 88,630 & 6846 & $\begin{array}{l}\text { Validated-by- } \\
\text { respondents (P2D) }\end{array}$ & 102,904 trips & $\begin{array}{l}2 \text { months collec- } \\
\text { tion period }\end{array}$ & Montreal & MTL Traject App \\
\hline $\begin{array}{l}\text { Yazdizadeh et al. } \\
\text { [122] }\end{array}$ & 88,630 & 6846 & $\begin{array}{l}\text { Validated-by- } \\
\text { respondents (P2D) }\end{array}$ & $\begin{array}{l}131,777 \text { trips } 33 \\
\text { mln GPS points }\end{array}$ & $\begin{array}{l}2 \text { months collec- } \\
\text { tion period }\end{array}$ & Montreal & MTL Traject App \\
\hline $\begin{array}{l}\text { Bohte and Maat } \\
{[21]}\end{array}$ & 40,208 & 1104 & $\begin{array}{l}\text { Validated-by- } \\
\text { respondents (P2D) }\end{array}$ & n.p. & 7395 days & Netherlands & $\begin{array}{l}\text { GPS logger and Web } \\
\text { based validation }\end{array}$ \\
\hline Thomas et al. [104] & n.p. & 600 & $\begin{array}{l}\text { Validated-by- } \\
\text { respondents }\end{array}$ & 60,000 trips & $\begin{array}{l}3 \text { batches per } 1 \\
\text { month each }\end{array}$ & Netherlands, [43] & Move smarter \\
\hline Xiao et al. [119] & 1248 & 202 & $\begin{array}{l}\text { Validated-by- } \\
\text { respondents }\end{array}$ & 4685 Trip-legs & n.p. & Shanghai & $\begin{array}{l}\text { Shangai City- } \\
\text { Smartphone based } \\
\text { travel survey }\end{array}$ \\
\hline Dabiri et al. [32] & 4000 & 189 & $\begin{array}{l}\text { Partially validated- } \\
\text { by-respondents ( } 69 \\
\text { respondents) }\end{array}$ & $\begin{array}{l}\text { 17,621 trajectories } \\
1,292,951 \mathrm{~km} \\
50,176 \mathrm{~h}\end{array}$ & $\begin{array}{l}3 \text { years collection } \\
\text { period }\end{array}$ & Beijing & Geolife [129] \\
\hline $\begin{array}{l}\text { Rasmussen et al. } \\
\text { [90] }\end{array}$ & 644 & 101 & $\begin{array}{l}\text { Validated-by- } \\
\text { respondents (P2P) }\end{array}$ & $\begin{array}{l}6,419,441 \mathrm{GPS} \\
\text { points } 1783 \mathrm{~h} \text { of } \\
\text { travel }\end{array}$ & $\begin{array}{l}3-5 \text { days per } \\
\text { respondent }\end{array}$ & Copenhagen & GPS logger \\
\hline Assemi et al. [11] & 372 & 76 & $\begin{array}{l}\text { Validated-by- } \\
\text { respondents }\end{array}$ & $\begin{array}{l}760,000 \text { GPS } \\
\text { observations, } 530 \mathrm{~h} \\
\text { trajectories }\end{array}$ & $\begin{array}{l}2 \text { months per } \\
\text { respondent }\end{array}$ & New-Zealand & $\begin{array}{l}\text { Advanced Travel } \\
\text { Logging Application } \\
\text { for Smartphones II } \\
\text { (ATLAS II) }\end{array}$ \\
\hline Mäenpää et al. [72] & 4000 & $>69$ & $\begin{array}{l}\text { Validated-by- } \\
\text { respondents }\end{array}$ & n.p. & n.p. & $\begin{array}{l}\text { Beijing. } 1 \text { week BUS } \\
\text { trajectories, } 1000 \\
\text { trajectories from } \\
\text { Open Street Map } \\
\text { (OSM) }\end{array}$ & $\begin{array}{l}\text { Geolife [129], } \\
\text { Journeys API }{ }^{a}, \\
\text { OpenStreetMap }^{b}\end{array}$ \\
\hline $\begin{array}{l}\text { Dabiri and Heaslip } \\
\text { [31] }\end{array}$ & 4000 & 69 & $\begin{array}{l}\text { Validated-by- } \\
\text { respondents }\end{array}$ & n.p. & $\begin{array}{l}3 \text { years collection } \\
\text { period }\end{array}$ & Beijing & Geolife [129] \\
\hline Jiang et al. [57] & 4000 & 69 & $\begin{array}{l}\text { Validated-by- } \\
\text { respondents }\end{array}$ & n.p. & $\begin{array}{l}3 \text { years collection } \\
\text { period }\end{array}$ & Beijing & Geolife [129] \\
\hline Zhou et al. [131] & 4000 & 69 & $\begin{array}{l}\text { Validated-by- } \\
\text { respondents }\end{array}$ & n.p. & $\begin{array}{l}3 \text { years collection } \\
\text { period }\end{array}$ & Beijing & Geolife [129] \\
\hline Zhu et al. [134] & 4000 & 69 & $\begin{array}{l}\text { Validated-by- } \\
\text { respondents }\end{array}$ & n.p. & $\begin{array}{l}3 \text { years collection } \\
\text { period }\end{array}$ & Beijing & Geolife [129] \\
\hline Zhou et al. [132] & n.p. & 12 & $\begin{array}{l}\text { Validated-by- } \\
\text { respondents }\end{array}$ & n.p. & $\begin{array}{l}6 \text { days per } \\
\text { respondent }\end{array}$ & Georgia (USA) & Self Developed App \\
\hline Martin et al. [75] & n.p. & 6 & $\begin{array}{l}\text { Validated-by- } \\
\text { respondents }\end{array}$ & $\begin{array}{l}347,719 \mathrm{GPS} \text { points } \\
\text { in } 96.59 \mathrm{~h}(1 \mathrm{~Hz}) 1.7 \\
\text { mln points Accel- } \\
\text { eration in } 98.62 \mathrm{~h} \\
(5 \mathrm{~Hz})\end{array}$ & n.p. & Minnesota & Self Developed App \\
\hline Byon and Liang [22] & n.p. & n.p. & n.p. & n.p. & $50 \mathrm{~h}$ & Toronto & Self Developed App \\
\hline $\begin{array}{l}\text { Jahangiri and Rakha } \\
\text { [55] }\end{array}$ & n.p. & n.p. & $\begin{array}{l}\text { Validated-by- } \\
\text { respondents }\end{array}$ & n.p. & n.p. & Tennessee & Self Developed App \\
\hline
\end{tabular}

a Journeys API, retrieved from web 01/01/2019, http://wiki.itsfactory.fi/index.php/Journeys_API

${ }^{b}$ Open-source Trajectories, retrieved from web 01/01/2019, https://www.openstreetmap.org/traces

process of data preparation, cleansing, and trajectory segmentation up front. We describe these processes in the next sections.
For each classifier, such as for mode detection and purpose imputation, the underlying features can be (i) location-agnostic versus location-specific; and (i) useragnostic versus user-specific. For example, methods 


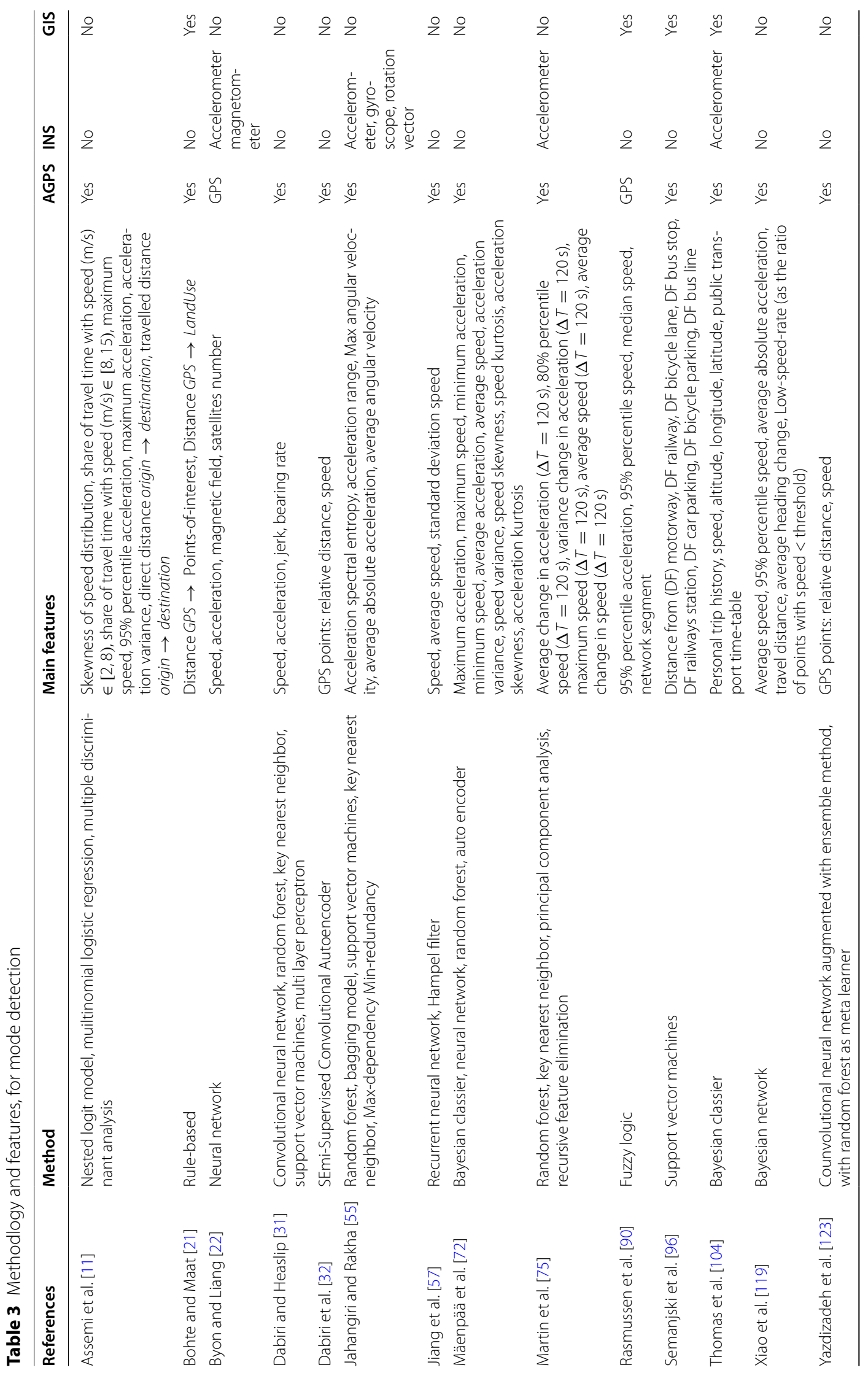




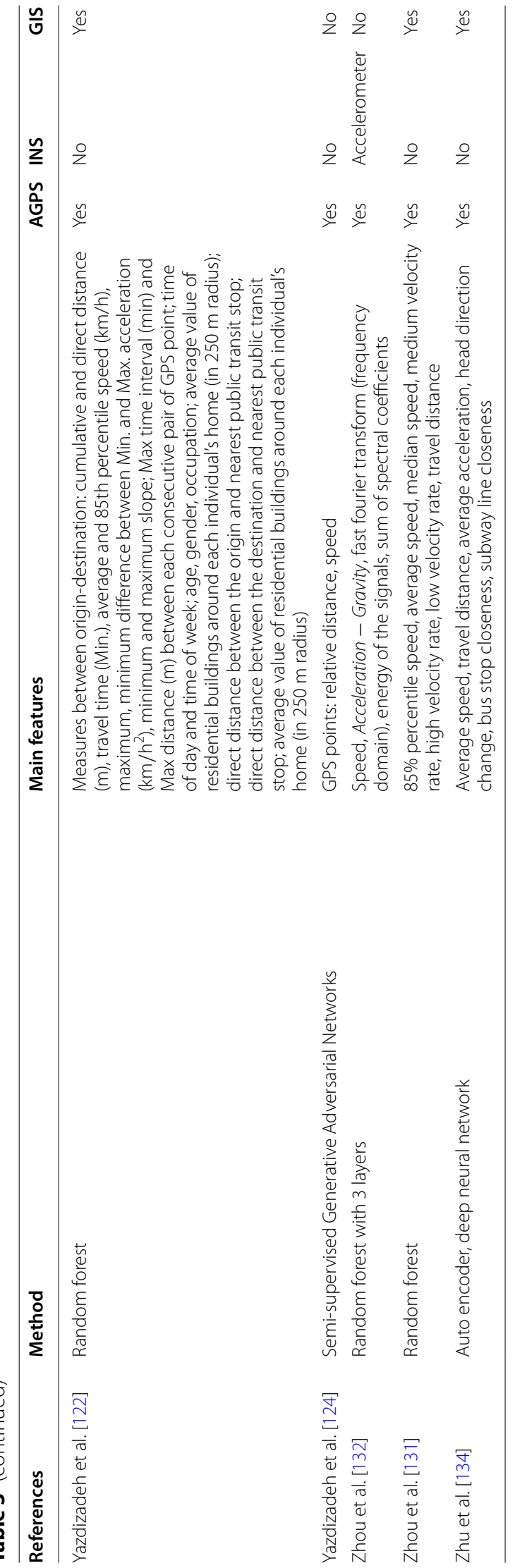


Table 4 Classification task ranked by difficulty and score, for purpose imputation

\begin{tabular}{|c|c|c|c|c|c|}
\hline References & No. & Classes & Score & Metric & Validation \\
\hline Kim et al. [60] & 15 & $\begin{array}{l}\text { Work, Study, Shopping, Social Visit, Recreation, Home, Business } \\
\text { Meeting, Change mode/Transfer, Pick-up, Drop-off, Meal/Eating } \\
\text { break, Personal Errand/Task, Medical/Dental, Entertainment, } \\
\text { Sport/Exercise }\end{array}$ & $98.68 \%$ & F1-Score & Out-of-bag-estimate \\
\hline Feng and Timmermans [40] & 10 & $\begin{array}{l}\text { Study, Social Visit, Recreation, Home, Service, Paid Work, Daily } \\
\text { Shopping, Non-daily Shopping, Help parents/cildren, Voluntary } \\
\text { work }\end{array}$ & $96.8 \%$ & Accuracy & Out-of-bag-estimate \\
\hline Montini et al. [76] & 9 & $\begin{array}{l}\text { Work, Shop, Service, Recreation, Home, Pick-up, Drop-off, Busi- } \\
\text { ness Meeting, Other }\end{array}$ & $79.8 \%$ & Accuracy & Out-of-bag-estimate \\
\hline Xiao et al. [120] & 8 & $\begin{array}{l}\text { Work, Study, Shop, Social Visit, Home, Eeating Out, Pick-up, } \\
\text { Drop-off }\end{array}$ & $96.53 \%$ & Accuracy & Hold-out \\
\hline Bohte and Maat [21] & 7 & Work, Study, Shop, Social Visit, Recreation, Home, Other & $43 \%$ & Accuracy & n.p. \\
\hline Yazdizadeh et al. [122] & 6 & Education, Health, Leisure, Shopping/Errands, Home, Work & $72 \%$ & $\begin{array}{l}\text { F1-Score } \\
\text { weighted } \\
\text { average }\end{array}$ & Manifold-cross-validation \\
\hline
\end{tabular}

relying on user- or location-agnostic features can be trained on any geographic area, and then either deployed on a different area to classify the activities of another population or reused to solve similar problems. The former depends on the generalization power of the model, while the latter is identified as transfer learning. Transfer learning is the discipline dedicated to using the knowledge gained by solving a problem in one domain (e.g. stop detection) to solve a different problem in another domain (e.g. mode and purpose classification). From our standpoint, these approaches could contribute in mitigating the cold-start problem [100], for example in the process of switching from a traditional to a smartphonebased travel survey.

The literature reviewed often works with location and user-agnostic features. In contrast, user- $[60,126]$ and location-specific [96] data seem to enable more accurate classifications. Although results presented in the relevant literature are hardly comparable across studies, within each relevant study we find evidence about the positive contribution of user- and location-specific data on the performance of the classifiers [104]. The cost is the volume of information to be handled, poor transferability and poor generalization power. From this angle, we challenge the conclusions of [62]: Transferability and generalization power may also be related to the supporting dataset, and not only to the machine-learning method.

\subsection{Data cleansing}

While performing data cleansing, data analysts should check whether basic features such as speed and acceleration are consistent with the context. The data cleansing purpose is to find and remove outliers, fill observation gaps, and possibly smooth the trajectories [1]. This crucial step should begin performing a sanity check on the observations' timestamps. Common issues are multiple observations with the same timestamp, or discrepancies due to implicit time localization that keeps no trace, e.g., of periodical solar and legal time shifts. The first case can be mitigated using fine grained timestamps during data collection, such as milliseconds or microseconds; the second, using standard date representations such as the ISO 8601. Further, sensors trajectories are often stored inconsistently on database, e.g., due to smartphones temporary lack of internet connection. Therefore, to find "correct outliers", any basic feature-such as speed, space, and time variation between consecutive pairs of observations-should be computed after sorting these trajectories by timestamp. Once the basic features are available, to handle outliers there are different degrees of sophistication between rule-based, statistical, and modelbased filters, such as threshold-, median-, and Kalmanfilter. The measurements' sampling rate is a critical factor determining the filter choice. In general, the trade-off is between scalability and accuracy, with rule-based filters on the one hand, and more sophisticated tools like the Kalman-filters on the other. If the number of outliers is very high, such that removing these outliers we create unacceptable gaps in the trajectories, data analysts can resort to one of the several data imputation techniques available [108], such as an exponential weighted moving average.

To reduce the risk of noisy labels that could bias supervised classifiers already in the training phase, data cleansing should focus on labels too. Often labels come as a separate trajectory, which should have a common timeline with the sensors' observations. We are aware that during the validation users may overlook errors present on travel diaries. We cannot exclude human-computer interaction problems facilitating 
Table 5 Dataset ranked by number of users, for purpose imputation

\begin{tabular}{|c|c|c|c|c|c|c|c|}
\hline References & Person-day & Users & Ground truth & Observations & Time & Area & Smart-phone App \\
\hline $\begin{array}{l}\text { Yazdizadeh et al. } \\
\text { [122] }\end{array}$ & 88,629 & 6845 & $\begin{array}{l}\text { Validated-by- } \\
\text { respondents (P2D) }\end{array}$ & $\begin{array}{l}\text { 131,777 trips, } 33 \\
\text { mln GPS points }\end{array}$ & $\begin{array}{l}1 \text { month collection } \\
\text { period }\end{array}$ & Montreal & MTL Traject App \\
\hline $\begin{array}{l}\text { Bohte and Maat } \\
\text { [21] }\end{array}$ & 40,208 & 1104 & $\begin{array}{l}\text { Validated-by- } \\
\text { respondents }\end{array}$ & n.p. & 7395 days & Netherlands & $\begin{array}{l}\text { GPS logger and Web } \\
\text { based validation }\end{array}$ \\
\hline Kim et al. [60] & 7856 & 793 & $\begin{array}{l}\text { Validated-by- } \\
\text { respondents (P2D) }\end{array}$ & $\begin{array}{l}22,170 \text { days, } 130 \\
\text { mln GPS points }\end{array}$ & $\begin{array}{l}5-14 \text { days per } \\
\text { respondent }\end{array}$ & Singapore & $\begin{array}{l}\text { Future Mobility } \\
\text { Survey }\end{array}$ \\
\hline $\begin{array}{l}\text { Feng and Timmer- } \\
\text { mans [40] }\end{array}$ & n.p. & 329 & $\begin{array}{l}\text { Validated-by- } \\
\text { respondents (P2D) }\end{array}$ & 10,545 activities & $\begin{array}{l}3 \text { month per } \\
\text { respondent }\end{array}$ & $\begin{array}{l}\text { Netherlands (Rot- } \\
\text { terdam) }\end{array}$ & $\begin{array}{l}\text { GPS logger and Web } \\
\text { based validation }\end{array}$ \\
\hline Xiao et al. [120] & 2409 & 321 & $\begin{array}{l}\text { Validated-by- } \\
\text { respondents (P2P) }\end{array}$ & 7039 trips & $\begin{array}{l}7-12 \text { days per } \\
\text { respondent }\end{array}$ & Shanghai & $\begin{array}{l}\text { Shangai City - Smart- } \\
\text { phone Based Travel } \\
\text { Survey }\end{array}$ \\
\hline Montini et al. [76] & n.p. & 156 & $\begin{array}{l}\text { Validated-by- } \\
\text { respondents }\end{array}$ & 6938 activities & 7 days & Zurich & Self Developed App \\
\hline
\end{tabular}

Table 6 Methodlogy and features, for purpose imputation

\begin{tabular}{|c|c|c|c|c|c|}
\hline References & Method & Main features & AGPS & INS & GIS \\
\hline Bohte and Maat [21] & Rule-based & $\begin{array}{l}\text { Distance GPS } \rightarrow \text { Points-of-interest, Distance } \\
\text { GPS } \rightarrow \text { LandUse }\end{array}$ & GPS & No & Yes \\
\hline Feng and Timmermans [40] & Random forest & $\begin{array}{l}\text { Activity duration, activity start time, } \\
\text { travel time to activity, distance } \\
\text { GPS } \rightarrow \text { Points-of-interest }\end{array}$ & GPS & No & Yes \\
\hline Kim et al. [60] & Bagging decision tree, random forest & $\begin{array}{l}\text { Activity probability, distance-based empiri- } \\
\text { cal probability, activity transition probability, } \\
\text { activity duration }\end{array}$ & Yes & Accelerometer & Yes \\
\hline Montini et al. [76] & Clustering, random forest & $\begin{array}{l}\text { start time, end time, GPS points density, } \\
\text { age, education, income, mobility ownership, } \\
\text { activity duration, walk percentage }\end{array}$ & Yes & Accelerometer & Yes \\
\hline Xiao et al. [120] & $\begin{array}{l}\text { Multi layer perceptron, particle swarm opti- } \\
\text { misation, multinomial logit, support vector } \\
\text { machines, Bayesian network }\end{array}$ & $\begin{array}{l}\text { Age, gender, education, working hours, } \\
\text { income, time of week, activity duration, } \\
\text { time of day, transportation mode, distance } \\
\text { GPS } \rightarrow \text { Points-of-interest, distance } \\
\text { GPS } \rightarrow \text { LandUse }\end{array}$ & Yes & No & \\
\hline Yazdizadeh et al. [122] & Random forest & $\begin{array}{l}\text { Features returned by Open Trip Planner }{ }^{\mathrm{a}} \\
\text { itinerary: GPS tracks average speed, time } \\
\text { interval between the first and last GPS track } \\
\text { of a trip, average distance between con- } \\
\text { secutive GPS point, attributes from, itinerary } \\
\text { length, total transit time of each returned, } \\
\text { total walking time of each itinerary, total } \\
\text { waiting time of each itinerary, total travel } \\
\text { time, number of transfers, walking distance, } \\
\text { itinerary average speed attributes from GPS } \\
\text { tracks, difference between GPS tracks length } \\
\text { and itinerary length, overlapping percent- } \\
\text { age of itinerary and GPS tracks }\end{array}$ & Yes & No & \\
\hline
\end{tabular}

${ }^{a}$ Open Trip Planner (OTP) retrieved from web 01/01/2019, https://github.com/opentripplanner/OpenTripPlanner

human errors during the travel diary validation step. Human errors may also occur while extracting data from the database. Rather than outliers, in these cases we should be concerned of flipping-labels [92]. Given a set of labels that a travel survey collects, outlyinglabels indicate one or more trajectories labeled with a class not included in this set; flipping-labels indicate one or more trajectories belonging to one class and labeled with another class, both being present in the set. However, while the impact of both outlying- and flipping-labels on supervised classifiers is extensively studied for independent and identical distributed data $[15,16,73,74,77]$-for example on the popular 
Table 7 Map-matching task ranked by difficulty and score

\begin{tabular}{|c|c|c|c|c|c|}
\hline References & Mode & Category & Score & Metric & Validation \\
\hline Chen and Bierlaire [26] & $\begin{array}{l}\text { Walk, Bike, Car, } \\
\text { Metro }\end{array}$ & $\begin{array}{l}\text { Multimodal, global, } \\
\text { shortest-path }\end{array}$ & {$[80 \%, 99 \%]$} & Path similarity indicator & n.p. \\
\hline Torre et al. [106] & Bicycle & $\begin{array}{l}\text { Match when possible, } \\
\text { build when needed }\end{array}$ & n.p. & n.p. & n.p. \\
\hline Quddus et al. [89] & Car & $\begin{array}{l}\text { Unimodal, incremental, } \\
\text { point-based }\end{array}$ & $99.2 \%$ & $A=\frac{\#(\text { correctly matched GPS points })}{\#(\text { Total GPS points })}$ & n.p. \\
\hline Li et al. [68] & Car & $\begin{array}{l}\text { Unimodal, incremental, } \\
\text { point-based }\end{array}$ & $\begin{array}{l}\text { 99.8\% (sub-urban), } \\
97.8 \% \text { (urban) }\end{array}$ & $A=\frac{\#(\text { correctly matched GPS points })}{\#(\text { Total GPS points })}$ & n.p. \\
\hline Wei et al. [115] & Car & $\begin{array}{l}\text { Unimodal, incremental, } \\
\text { shortest-path }\end{array}$ & $98 \%$ & Accuracy & n.p. \\
\hline Bierlaire et al. [19] & n.p. & $\begin{array}{l}\text { Unimodal, global, } \\
\text { shortest-path }\end{array}$ & {$[80 \%, 99 \%]$} & Path similarity indicator & n.p. \\
\hline Wu et al. [116] & Taxi & $\begin{array}{l}\text { Unimodal, incremental, } \\
\text { point-based }\end{array}$ & $93.58 \%$ & $\begin{array}{l}\text { Prediction accuracy of next road } \\
\text { by the road having the maximum } \\
\text { probability }\end{array}$ & Hold-out \\
\hline Hunter et al. [52] & Taxi & $\begin{array}{l}\text { Unimodal, incremental, } \\
\text { shortest-path, supervised, } \\
\text { unsupervised }\end{array}$ & $\begin{array}{l}100 \% \text { (1 s resolution), } \\
>90 \% \text { (30 s resolution) }\end{array}$ & Accuracy & $\begin{array}{l}\text { Manifold- } \\
\text { cross- } \\
\text { validation }\end{array}$ \\
\hline Li and Wu [67] & Taxi & $\begin{array}{l}\text { Unimodal, incremental, } \\
\text { point-based }\end{array}$ & $87.18 \%$ & $A=\frac{\#(\text { correctly matched GPS points) }}{\#(\text { TotalGPS points) }}$ & Hold-out \\
\hline $\begin{array}{l}\text { Jagadeesh and Srikan- } \\
\text { than [54] }\end{array}$ & $\begin{array}{l}\text { Dataset 1: Taxi. } \\
\text { Dataset 2: n.p. }\end{array}$ & $\begin{array}{l}\text { Unimodal, global, } \\
\text { shortest-path }\end{array}$ & $91.3 \%$ & $\begin{array}{l}\text { Average F-Score with: } \\
\text { Precision }=\frac{\text { Length }_{\text {correct }}}{\text { Length }_{\text {matched }}} \\
\text { Recall }=\frac{\text { Length }}{\text { Leorrect }}, \text { Input-to-output } \\
\text { latency (Timelines) }\end{array}$ & Hold-out \\
\hline Newson and Krumm [78] & Car & $\begin{array}{l}\text { Unimodal, incremental, } \\
\text { point-based }\end{array}$ & $\begin{array}{l}\text { 100\% ( } 1 \text { s resolution), } \\
>90 \% \text { (30 s resolution) }\end{array}$ & $\begin{array}{l}\text { Accuracy }=1-E_{L}, \text { where } \\
E_{L}=\frac{\left(d_{-}+d_{+}\right)}{\left(d_{0}\right)}, d_{-}=\text {erroneous } \\
\text { subtracted length, } d_{+}=\text {erroneous } \\
\text { added length, } d_{0}=\text { length of correct } \\
\text { route }\end{array}$ & Hold-out \\
\hline Lou et al. [71] & n.p. & $\begin{array}{l}\text { Unimodal, global, } \\
\text { shortest-path }\end{array}$ & $A_{N}>81 \%, A_{L}>87 \%$ & $\begin{array}{l}A_{N}=\frac{\#(\text { correctly matched road segments })}{\#(\text { all road segments of the trajectory })} \\
A_{L}=\frac{(\Sigma \text { length of matched road segments })}{(\text { length of the trajectory })}\end{array}$ & Hold-out \\
\hline
\end{tabular}

handwritten digits dataset from the Modified National Institute of Standards and Technology databasewe found no literature focusing on time series, as for example GPS.

\subsection{Trajectory stop detection}

The analysis of human trajectories can be reduced to two fundamental classes: motion, and stop. Tables 1 and 4 present how each class branches out. Tables 3 and 6 specify both features and methods enabling accurate classifications. Tables 2 and 5 present the dataset that enabled each study we reviewed. To perform any specialized inference on trip legs we need to identify homogeneous segments and relevant discontinuities from heterogeneous and complex mode-chain-types.

A GPS segment is considered a stop candidate if it lays within a topologically closed polygon for a certain time $[4,128,133]$. The presence of GPS points nearby may be indicative of a stop-the absence of motion [107]. Rules to acquire a local density of points, for example, include a moving window linking 30 preceding and 30 succeeding points within a $15 \mathrm{~m}$ range [94]. Although compatible with the error amplitude of GPS devices declared in a survey by [37], this range seems too small compared to smartphone AGPS expected error [109]. Smartphones location output does not rely exclusively on GPS, but also on less accurate methods that fill GPS gaps. Zhao et al. [126], for example, extend the range to $45 \mathrm{~m}$.

Based on the assumption that noise detected in transition points is temporary while the changes in speed are permanent, affinity propagation clustering methods can be effective in stop detection [133]. By building a network that links stationary events, identified as nodes within a critical space-time range, and clustering this network 


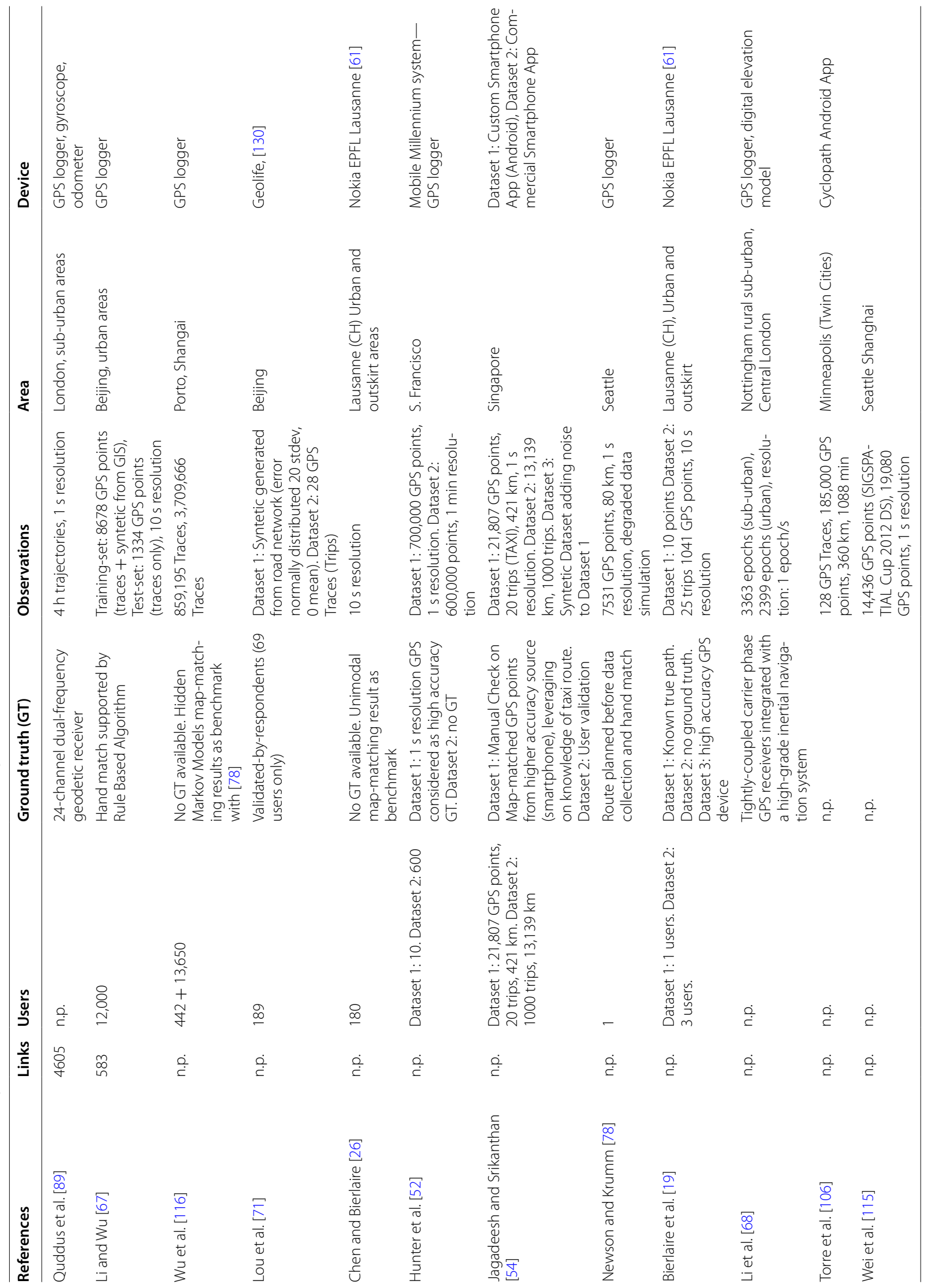


using two-level Infomap [93], a swift algorithm, available as python package [9], outputs a label for each stop event detected in a raw GPS trajectory.

Literature shows many developments in this direction, employing clustering techniques $[46,105,117,130]$, which can learn in an unsupervised fashion and find stops within GPS trajectories. In multiple-step approaches, personal- [46], and geographical-context [130] can augment trajectories' information and improve the classification of stop candidates. Density-based spatial clustering of applications with noise (DBSCAN) is at the base of most frameworks; some of these frameworks can even find stop candidates directly on raster image representations [111]. Many other effective probabilistic unsupervised methods are available, as for example kernel-based [48, 103]; generative [81, 118]; and discriminative [70], such as kernel-density algorithms, Hidden Markov Models, and conditional random fields.

Assuming that travelers walk to change mode, a rulebased algorithm can identify transition points by applying thresholds on speed, acceleration, range and time, as well as by checking GPS on-off status [90]. In fact, the most common rule-based stop detection techniques rely on range, time, speed or acceleration thresholds [98].

These rule-based algorithms can be further improved by statistical tests. For example, a Kolmogorov-Smirnov test on a random sample can be used to check for outliers [131], as the normal distribution is sometimes accepted as a suitable approximation for GPS. Assuming normal distribution of GPS error, though, GPS follows a bi-variate Raleigh distribution [19].

Rule-based algorithms are both effective and appropriate, and are independent of the subsequent classification task, as for example mode detection, or purpose imputation. However, thresholds inflexibility (for example, in handling GPS signal loss and signal noise) leads to poor performance in detecting short stops (such as alighting from a bus) and long permanence in the same position (such as sitting on the bus during and intermediate stop) [98].

\subsection{Trajectory segmentation}

Another approach specialized in "mode detection" is a GPS trajectory preparation through segmentation, which goes through four steps [31]. The first step splits the trajectory in fixed segments having the same size of the median number of points on all the available trips. The second step concatenates together consecutive segments with the same label. Let us note that the first two steps depend strictly on the availability of the ground truth, while the segment size depends on the data collection context. The third step discards segments with less than
10 GPS points. The fourth step smooths the trajectory through a Savitzky-Golay filter.

Segmentation methods can be distance-, time-, bearing- and window-based. While the last three are statistically equivalent, the first leads to varying sample sizes within each segment due to the different speeds in complex mode-chain-types. Discontinuities in the mode-chain-type, detected on these segments, represent stops [57].

The impact of stop-detection or trip segmentation on the quality of the travel diary generation process, and therefore on the quality of the ground truth collected from users that validate their trips, can be considerable [102]. Therefore, more advanced hybrid methods have been studied, as have multiple rules and machine-learning specializing in both trajectories and contexts. One hybrid method consists of the following six steps [126]: The first step is trajectory cleansing, based on the accuracy provided by the AGPS; the second step is rule-based detection of stop candidates, where stops are points within a 50-m range and a 1-min time window. The third step checks for stop candidates against users' frequent stop locations. The fourth step merges the resulting stops, with a rule-based algorithm configured with various range and time thresholds. The fifth step detects "still" mode, with a learned classifier based on acceleration. The sixth step removes, after mode detection, any orphan stop left.

\subsection{Towards a standardized measurement of performance}

All of the aforementioned methods are very critical for the classification steps downstream in the process, and they all lack of flexibility in adapting to different thresholds, which might depend on some users, context, or both. However, the choice of trip segmentation method determines the object to be classified in the next step of the process, which can be a single observation, such as a GPS point, or a set of observations, such as a GPS segment. Consequently, two methods presenting the same classification score might be very different, depending on whether these methods target points or segments. It is very unlikely that the same number of points and segments will identify two analogous trips in terms of space and time. Therefore, comparing the performance score between point- and segment-based methods is misleading. The scores presented in Tables 1, 4 and 7 are not comparable, nor harmonized. Since scores and respective results reflect the case of correct classifications related, e.g., to a stage, a trip, an excursion or the whole day, harmonization attempts should take these cases explicitly into account.

Prelipcean et al. [86] introduce penalty systems and metrics that look at where these methods lead to errors, 


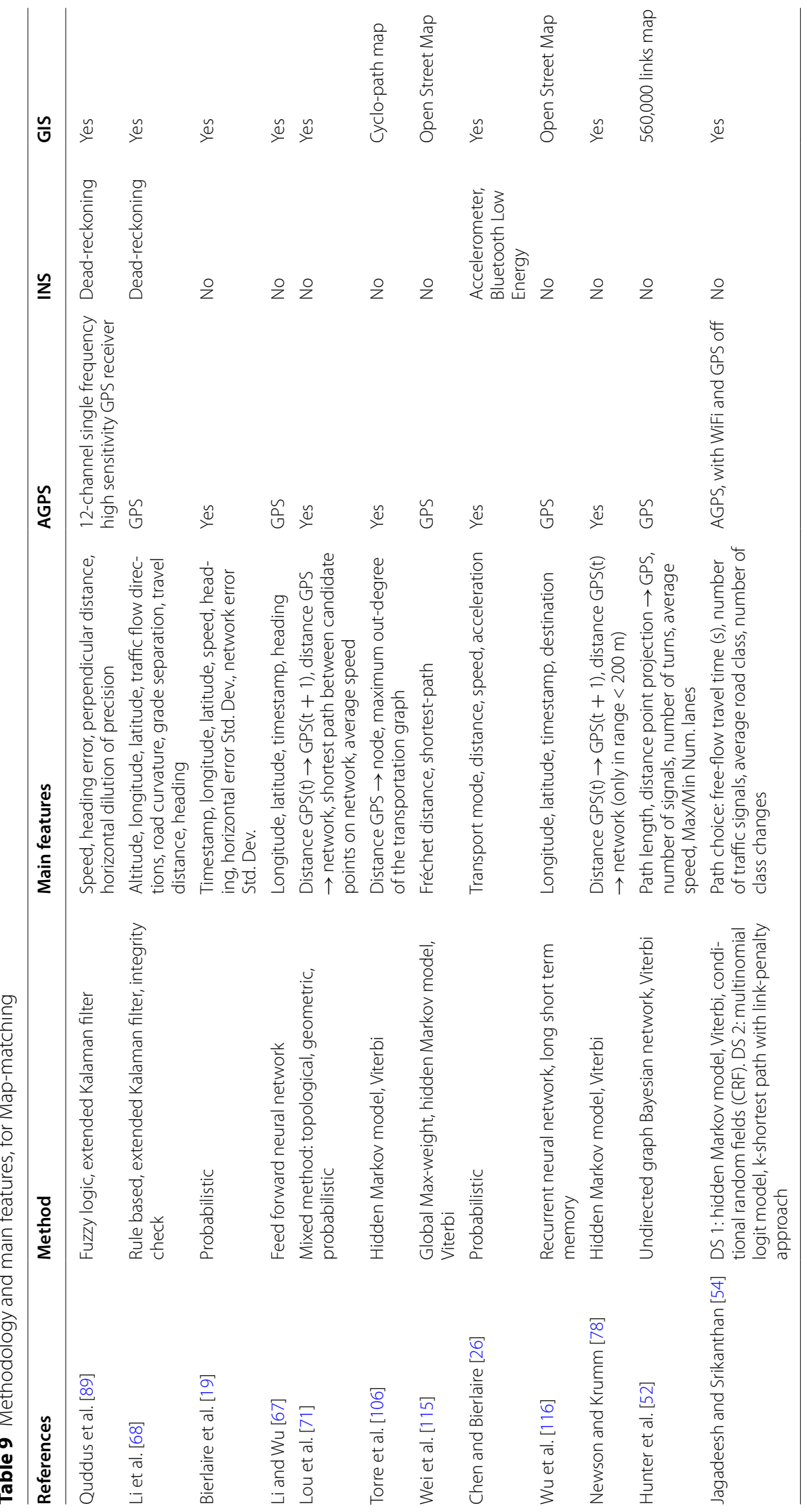




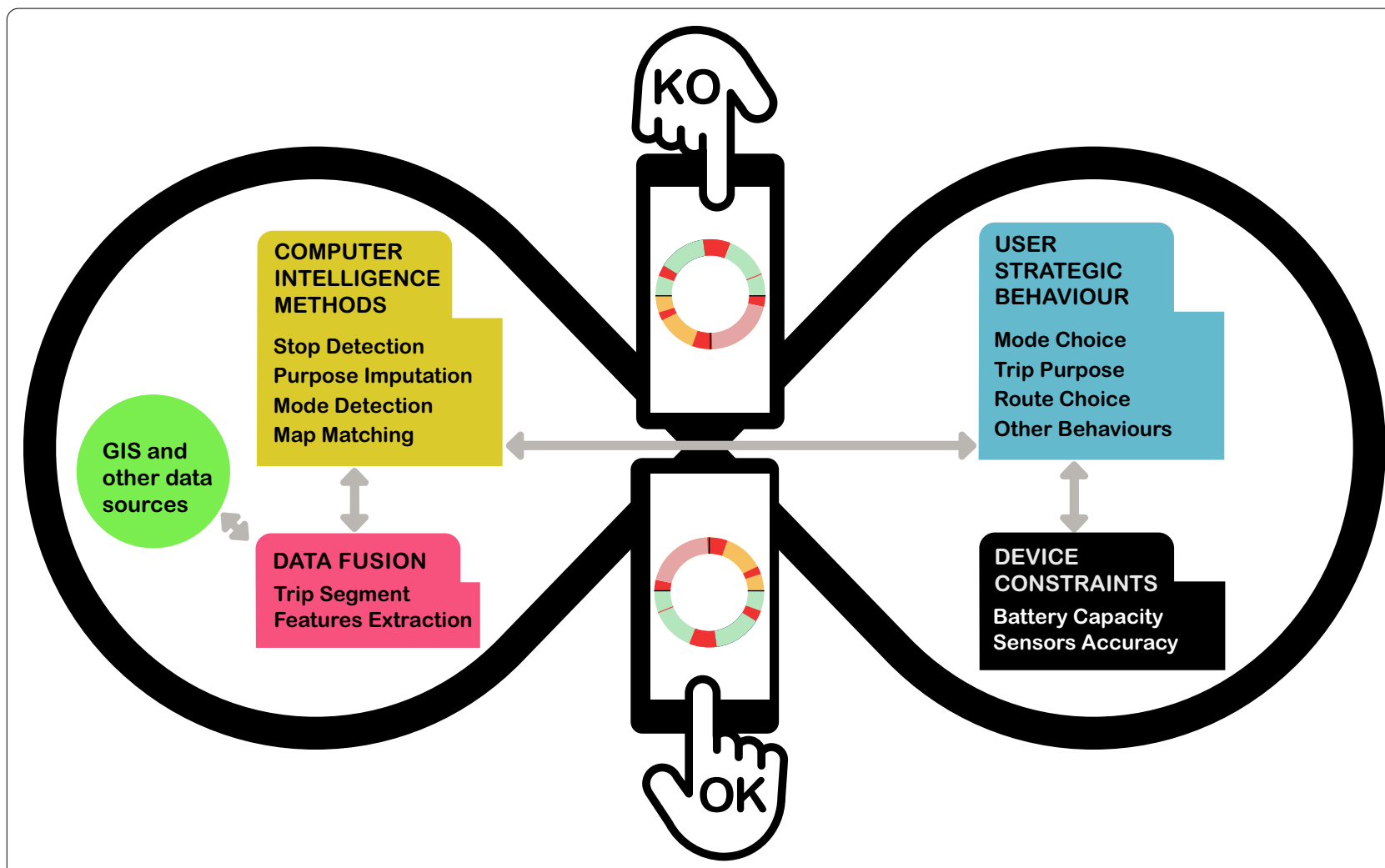

Fig. 3 Validation loop in smartphone-based user activity monitoring

and provide meaning to the comparison among different segmentation techniques. In particular, with respect to the ground truth, if precision and recall identify "hits" and "misses" of a classifier (the broadly used F1-Score is the harmonic mean of precision and recall) from such measurements, we do not understand how the error depends on over- or under-segmentation, e.g., of the trajectory that this method classified. Since errors in trajectory segmentation propagate to the classification of the trajectories, and classification performance depends on how the segmentation inference aligns with the ground truth, these penalties are proportional to time and space of segments misaligned with the ground truth. This is in opposition to previous studies where a count of the editing operations was proposed [2]. Interestingly, with this metric, point-based trajectory segmentation techniques seem to outperform segment-based techniques [86]. Since both segment- and point-based classifiers discard any segment below a certain threshold of (e.g.) GPS observations-which in the first case can be two magnitudes higher than in the second case-an intuitive explanation is that segment-based classifiers are incapable of classifying a larger fraction of a dataset.

\subsection{Human activity recognition in mobility}

To support the modeling of activity and travel choices at the heart, for example, of activity-based models [110], human activity recognition in mobility must include both stop, mode and purpose of any trip. The combination of feature extraction techniques and computer intelligence algorithms allows for a capturing of the correlation between features and the user's strategic choices. As technology evolves, the inference of users' strategic choices in the form of a travel-diary and user validation by means of such a diary (see Fig. 3), enable continuous improvement of the acceptable truth asymptotically approaching the theoretical ground truth. Computer intelligence algorithms are tightly coupled with the data necessary to allow and refine the inferences. Given an initial validated dataset, their performance can be measured only by comparing inferences with the ground truth (see Fig. 3). Errors propagate from trajectory segmentation, to trajectory classification, and then to the travel-diary generation [86]. Therefore, it is likely that errors propagate to the ground truth. From this standpoint, the output of this process might lead to systematically biased predictions. In SBTS, machine-learning is just a tool used to capture the information represented by data. The quality of models has a strong influence on the quality of the 
ground truth we can collect through travel-diaries, and vice-versa.

There is consensus in the field about the lack of standardization for validating and comparing competing classifiers. There are several studies where, even though classifications are performed on the same dataset, differences in number and quality of classes predicted and in validation setup are enough to make F1-Score comparisons meaningless. For example, F1-Scores obtained as average on a 5-label transport mode classification task and a fivefold cross-validation [31], cannot be compared with F1-Scores from a 4-label transport modes classification task, computed on a random test-set only (hold-out method) [57].

We have identified three approaches that allow for a comparison to be made between different methods and datasets. The first is the same aforementioned penalization solution to ease the comparison between point- and segment-based classifiers [86]. The second approach could provide a standardized baseline by combining a public dataset and a cross-validation workflow [112]. The dataset includes the observations of 18 sensors on three users made over a period of 2812 h' worth of labeled data. Labels include the position of the phone as: in the hand, at the torso, at the hip, and in a bag. The workflow for cross-validation covers three tasks: user-independent, phone position-independent, and time-invariant. At the end of the three tasks, each one accomplished with manifold cross-validation, the paper suggests the standard deviation of F1-Scores computed across users, phone positions, and time periods as the benchmark of the predictive power of a model. This workflow cannot be applied in most of the datasets available, which are not as rich; for example, the widely used Geolife [129] provides GPS trajectories and transport mode labels only (see Table 1). The third approach leverages the Weka software [47], where several machine-learning algorithms are available off-the-shelf. Based on Weka software, Ectors et al. [36] compare a few rule-based and probabilistic machine-learning algorithms for purpose imputation on the same dataset.

However, we found no attempts at combining these three approaches, which are complementary to comparing different methods, but not self-sufficient. Another step should consider the feature extraction process. Indeed, this process is also subject to attempts of standardization. One candidate method is "minimum redundancy maximum relevance" [112] (MRMR, see Table 3). For classifiers relying on deep learning though, this feature extraction method is not effective, as the neural network extracts the features autonomously. In this case, the new challenge is finding optimal hyper parameters for the neural network. Such hyper parameters may include, for example, architecture configuration, activation functions, batch size, regularization factor, and optimization step. Balaprakash et al. [14] propose an approach to selecting these hyper parameters automatically, moving towards standardized deep learning method optimization. Still, we did not find applications in this field; instead, optimal hyper parameters are still a craftsman product [31, $57,121]$.

\subsection{Implications for transport science}

The choice of complementary sensors, such as the gyroscope, could mitigate the challenges that most of the algorithms encounter in discriminating between, for example, bike and walk or bike and bus in congested urban contexts. Similarly, the magnetometer could help distinguish between rails and cars, and the accelerometer between bike and e-bike. However, these high-frequency sensors require online rather than offline classifiers. Offline classifiers would suffer from the large footprint of the data, which would in turn have a negative impact on smartphone users' data plan and battery. This would ultimately lead users to dropout from travel surveys.

Several studies exhibit how useful GIS information can be on mode detection. However, when classifying the complement of the same trajectory, studies on purpose imputation expose the challenges associated with the proximity of heterogeneous points of interest, as various trips can start for different purposes and end in the same spatial range. In such a case generally helpful, personal patterns and a limited amount of personal information proved to support more accurate predictions (see Table 3 against Table 1, and Table 6 against Table 4).

Nevertheless, among the studies identified for mapmatching, we find no examples of personal information use (see Table 9). Even in the assumption of unavailability of any personal information, map-matching and consequent route-choice records would amplify the impact of transport mode and trip purpose classification (see Table 7). Expressing a trajectory as a sequence of links and nodes on the transport network, instead of longitude and latitude, pinpoints specific micropatterns. Furthermore, it potentially reduces the confusion that users often face while validating their travel-diaries in the presence of GPS outliers.

For map-matching, we identify two problems. First, most of the methods specialize in cars and road network for cars, and few or none refer to emerging modes such as e-bikes and e-scooters (see Table 8). Second, in the literature, we did not find a good representation of adequate datasets and ground truth quality levels (see Table 9). In the first case, the assumption that GPS points should 
belong to the road network does not hold. Map-matching for modes different from cars requires degrees of freedom to allow transit on, for example, sidewalks and bicycle lanes, often not mapped-few studies pinpoint this problem. In contrast, emerging shared modes such as e-bikes and e-scooters imply behaviors not strictly coherent with the mapped network. Furthermore, these emerging modes are introducing new public transport mode-chain-types with irregular patterns, alternating traditional public transport and emerging shared modes. The former offers reliable timetables, while the latter is volatile, as it depends on vehicle availability. Still, Sicotte et al. [99] show that looking at meaningful mode-chaintypes also represent a tool to improve trip classification.

From the direct experience testing Mobile Market Monitor and TRAVELVU on a small user base, we realize that the sample of literature reviewed in this work does not express the differences between a raw trajectory, such as the one that SBTS use to generate travel-diaries, and a processed trajectory, such as the one that SBTS may output as ground truth. The first trajectory presents a level of noise that could even ease trip segmentation process and subsequent classification on uni-modal segments. The lack of noise of in the second trajectory, in contrast, might prevent accurate travel-diary generation. These obvious differences have an impact on the choice of method and performance of any transport-related analysis, such as for mode detection. For example, we expect better generalization of Bayesian temporal models or artificial neural network methods in the first case, and machine-learning techniques such as random forest or support vector machines in the second case.

Further, Tables 3, 6, and 9 clearly show that while artificial neural networks and temporal models do not require particular feature extraction methods, machinelearning approaches such as random forest or support vector machines must rely on time-series feature extraction. Hence, to find the best classification method, e.g. for transport mode, any attempt at ranking should be considered in light of whether the trajectories of interest embody any pre-processing, and possibly which one. A possible indicator is the proportion of point loss on the dataset after the application of simple filters, e.g. on point speed and time gaps between points.

For travel-diary generation in presence of multiple sensors and large datasets, artificial neural networks seem very promising. Artificial neural networks are flexible in learning with and without labels. They also act as powerful dimensionality-reduction, information-compression, and feature-extraction tools for simultaneous signal processing of multiple sensors monitoring the same event, and signaling at different and irregular frequencies. Let us consider, for example: (i) smartwatches and other bio-metric devices complementary to smartphones [29]; (ii) ongoing software integration between cars and smartphones, which include navigation and INS sensors [8]; and (iii) development of edge-computing to augment the processing power of smartphones when consuming cloud services [113], where users' mobility patterns are studied to reduce service-latency in the information-technology-network.

A holistic approach could amplify the impact of studies sharing the scope of those identified in this review. Smartphones' onboard sensors represent only a fraction of the collectible signals, and the surveyed literature seem not fully aware the quickly-evolving context surrounding smartphone devices. To release new potential towards the disambiguation of transport patterns that in congested urban areas look exactly the same for the surveyed methods, while contrasting the curse of dimensionailty [17], this field requires a new perspective. Compared to the advances in other fields, such as computer vision or social networks, transport science seems only at the beginning of the exploration of artificial neural networks .

\section{Discussion}

SBTS depends on a sophisticated multi-sided platform which is subject to often conflicting interests over the resources available, beginning with the battery. In current versions, the OS orchestrates the applications' use of sensors and battery, and some OS preclude direct access to AGPS. Therefore, developers have limited configuration possibilities. Furthermore, the data collected through these platforms is affected by large standard deviation, severe errors, and noise due to exogenous elements.

\subsection{Sensors}

When a smartphone outputs a location signal, whether the location comes from the onboard AGPS, from the triangulation with GSM antennas, the car GPS, or another external GPS connected to the smartphone, developers are not allowed to know. If not properly handled, this uncertainty may negatively affect datasets, method classification performance, user validation and finally ground truth.

Smartphone onboard sensors represent only a fraction of the bio-metric and ambient sensors that could be connected with these devices. Cornacchia et al. [29] present a survey of activity classification from wearable sensors. Differing effective frequencies of each sensor, e.g., $1-10 \mathrm{~Hz}$ for GPS, or $>20 \mathrm{~Hz}$ for accelerometer, require flexible frameworks as for joint features extraction, compression, and analysis. From this standpoint, artificial neural networks seem to have potential. 


\subsection{Data sources}

From the perspective of smartphone-related trajectories, a better understanding of travel behavior requires the standardization of measures relevant for travel patterns, which should also rely on standard datasets. The options available are a good starting point, but still seem insufficient. For example, let us consider the following datasets. (i) Shankari et al. [97] deliver real GPS trajectories collected in the USA from real smartphones, in which ground truth, available on trip mode and not trip purpose, is generated synthetically to protect privacy exposure (users follow instructions provided by a custom App). (ii) Wang et al. [112] offer trajectories collected in the UK from multiple smartphone sensors at relevant frequencies, and from smartphones of the same model positioned on various part of the body, providing ground truth for trip mode only. (iii) Zheng and Fu [129] include GPS trajectories from China, with ground truth on trip mode for 69 users out of 189. (iv) Kubicka et al. [63] supply GPS trajectories collected in various parts of the world for map-matching, but not multi-modal. (v) Carpineti et al. [24] propose onboard high-frequency sensors with ground truth on transport mode, collected in Italy from multiple smartphones and users, but where GPS is unavailable. (vi) Chavarriaga et al. [25] provide data from over 72 wearable sensors, collected indoors with ground truth on performed activities, and no GPS. (vii) Laurila et al. [65] offer data collected in Switzerland over 18 months from 185 users of the Nokia N95 device with multiple sensors, including, for example, AGPS, accelerometer, Bluetooth, trip purpose labels, and no transport modes.

\subsection{Methods}

The collection of any acceptable ground truth depends on the reliability and accuracy of underlying measurement methods. The vast choice of alternatives requires a standardized way of comparing competing methods. Existing literature offers effective penalization systems for classic performance scores [87]. Invitations on standardized mode detection are available in form of feature extraction and cross-validation workflows [112]. However, these attempts do not seem sufficient to cover mode detection, purpose imputation, and map-matching at the same time across existing and emerging methodologies.

We identified excellent alternatives. Some perform best on low-resolution trajectories. Other classifiers are tight (e.g.) to the location where GPS trajectories are fused with data from GIS, users' personal information, or both. Among the best performers in terms of accuracy measurement, in general, we find: support vector machines, fuzzy logic, random forests, and probabilistic models (e.g., hidden Markov models). Classic rule-based algorithms might not perform at the same accuracy level. However, they are still competitive when the application scenario is stable, and if execution speed and scalability are a priority over accuracy.

Methods based on artificial neural networks are rising quickly and are applicable across mode detection, purpose imputation, and map-matching, as probabilistic and Bayesian methods unlike other machine-learning techniques. For map-matching and purpose imputation, for example, we find applications combining GPS and GIS, while for stop and mode detection, we find applications with GPS only. Particular configurations of these methods, such as variational auto encoders and deep kalman filters, which represent the convergence with Bayesian methods, could offer a background facilitating methodological convergence that might also allow for a breakthrough in this mature field of research.

\subsection{Ground truth}

Whether a study targets, for instance, the whole day, week, month, season or year, modelers need a correct dataset ideally of a whole period. If this is not the case, the value of the whole dataset is limited. Since a "person to device" validation might introduce further errors; their magnitude and their impact on machine-learning methods performance should be investigated. We find no attempt of self-learning on multi-sensor datasets, which would raise expectations on a "device-to-device" ground truth evolution. We could achieve full automation of both travel-diary generation and validation by using independent measurements of the same event to substitute traditional labels with pseudo-labels. For example, instead of learning from labels, artificial neural networks could learn GPS patters to reconstruct accelerometer patterns, and vice-versa. Meanwhile, where machine-learning algorithms do not provide correct travel-diaries to the user, "person to device" interaction could be enhanced by introducing the possibility for the user: (i) to trigger a specialized automatic evaluation of such segments; and (ii) to flag whether he or she was unable to correct the mistakes (see Fig. 3).

\section{Conclusion}

In transport science, the process of methodological perfection between paper-and-pencil personal interviews, and computer assisted personal interviews [13], towards computer assisted telephone interviews [79], and computer assisted web interviews [135] is still evolving towards SBTS [101, 127]. The leap between paper and computer determined a structural impact on the surveying costs, requiring software, IT-infrastructure, and personnel-training. According to [27], the shift to computer assisted web interviews requires to fall back to 
telephone interviews in cases where the web interviews are incomplete.

From computer to smartphones, the impact seems negligible both on software and IT-infrastructure costs. In contrast, the impact on human resources seems to determine a significant reduction of personnel, and a shift towards highly specialized and more expensive skills of data scientists required to deploy a SBTS. Consequently, under a certain volume-threshold of, e.g., surveyed users in time, traditional surveys could be still competitive in terms of cost. However, to push transport science boundaries under the constraint of Big Data-which traditional travel surveys are unable to satisfy-SBTS bring a huge scalability potential and support higher resolution datasets, handling users during time horizons longer than just one day.

To expose SBTS potential, this paper selects and summarizes information on SBTS relevant for a qualitative comparison of the methods focusing on mode detection, purpose imputation, and map-matching. To ease such a comparison, since the standardization process in the field is still ongoing, we organized the literature into tables, which include information about classification objectives, datasets employed in the experiments, and validation approach of both data and experiments. Besides, by listing sensors, features, and dataset that each of the related works depends on, we identify the main methods underlying the process of ground truth generation.

Comparison based only on scores reflecting different variables, such as accuracy and F-Score, is misleading. As we find, scores depend on the underlying dataset, trajectory segmentation, classification method and experiment design. Evaluation of larger segment units leads to discarding significant portions of a dataset. The classification task is relatively more difficult with a larger number of classes. The accuracy bias is relatively lower when performing cross-validation, and when processing more representative datasets. For example, Tables 1 and 2 for mode detection, Tables 4 and 5 for purpose imputation, as well as Tables 7 and 8 for map-matching expose, from another perspective than Prelipcean et al. [86], that methods performance is beyond dry scores. When comparing methods, newcomers in this field would certainly benefit from considering task complexity, representativeness of the supporting dataset, and validation method. For example, task and method complexity, features collection and extraction cost (see Tables 3, 6, 9).

A converging thrust in the field seems represented by simultaneous methods focusing on, e.g., mode detection to improve map-matching or purpose imputation, and vice-versa. To support the disambiguation of travel patterns that are still challenging to detect in congested urban areas, for the future, emerging applications of artificial neural networks seem to support further fruitful convergence. The study of smartphones onboard sensors in addition to other streams collectible through smartphones-from GIS, wearable sensors, or edge-computing-would benefit from the artificial neural networks flexible framework. This technology can be exploited on the one hand to learn from large and heterogeneous data streams, and on the other hand to compress and store such BIG bulk of information through relatively few trained parameters. To support the standardization of relevant measures for transport behavior, efforts should also be directed towards the solution of privacy concerns that represent an obstacle, in this field, for the generation of open-access datasets.

\section{Abbreviations}

AGPS: Assisted global positioning systems; CPU: Central processing unit; GIS: Geographic information systems; GPS: Global positioning systems; GPU: Graphical processing unit; INS: Inertial navigation systems; OS: Operation systems; P2D: Person-to-device; P2P: Person-to-person; SBTS: Smartphonebased travel surveys.

\section{Acknowledgements}

Not applicable.

\section{Authors' contributions}

VS: Conceptualization, methodology, data curation, investigation, formal analysis, writing —original draft. FCP: Conceptualization, validation, supervision. MKA: Validation, editing. OAN: Conceptualization, validation, supervision. All authors read and approved the final manuscript.

\section{Funding}

Not applicable.

Availability of data and materials

Not applicable.

Declaration

\section{Competing interests}

The authors declare that they have no competing interests.

Received: 27 November 2020 Accepted: 20 October 2021

Published online: 06 November 2021

\footnotetext{
References

1. Abbruzzo, A., Ferrante, M., \& Cantis, S. D. (2021). A pre-processing and network analysis of GPS tracking data. Spatial Economic Analysis, 16(2), 217-240.

2. Allen, J. F. (1983). Maintaining knowledge about temporal intervals. Communications of the ACM, 26(11), 832-843.

3. Allström, A., Kristoffersson, I., \& Susilo, Y. (2017). Smartphone based based travel diary collection: Experiences from a field trial in Stockholm. Transportation Research Procedia, 26, 32-38.

4. Alvares, L. O., Bogorny, V., Kuijpers, B., De Macedo, J. A. F., Moelans, B., \& Vaisman, A. (2007). A model for enriching trajectories with semantic geographical information. In GIS: Proceedings of the ACM international symposium on advances in geographic information systems.

5. Anderson, P., Hepworth, M., Kelly, B., \& Metcalfe, R. (2007). What is Web 2.0 ? Ideas, technologies and implications for education by. Technology, $60(1), 64$.

6. Apple. (2016). Apple developers support resolution on network signal strength access. Retrieved January 1, 2019, from web.
} 
7. Apple. (2019). Preventing unexpected shutdowns. Retrieved January 1 , 2020, from web.

8. Apple. (2021). Car data integration on smartphones. Retrieved March 17, 2021, from web.

9. Aslak, U. (2019). Infostop, a Python package for detecting stop locations in mobility data. Retrieved November 26, 2019, from web.

10. Assemi, B., Jafarzadeh, H., Mesbah, M., \& Hickman, M. (2018). Participants' perceptions of smartphone travel surveys. Transportation Research Part F: Traffic Psychology and Behaviour, 54, 338-348.

11. Assemi, B., Safi, H., Mesbah, M., \& Ferreira, L. (2016). Developing and validating a statistical model for travel mode identification on smartphones. IEEE Transactions on Intelligent Transportation Systems, 17(7), 1920-1931.

12. Auld, J., Williams, C., Mohammadian, A., \& Nelson, P. (2009). An automated GPS-based prompted recall survey with learning algorithms. Transportation Letters, 1, 59-79.

13. Baker, R. P., Bradburn, N. M., \& Johnson, R. A. (1995). Computer-assisted personal interviewing: An experimental evaluation of data quality and cost. Journal of Official Statistics, 11(4), 413-431.

14. Balaprakash, P., Salim, M., Uram, T. D., Vishwanath, V., \& Wild, S. M. (2019). DeepHyper: Asynchronous hyperparameter search for deep neural networks. In Proceedings-25th IEEE international conference on high performance computing, HiPC 2018 (pp. 42-51).

15. Barandela, R., \& Gasca, E. (2000). Decontamination of training samples for supervised pattern recognition methods. In F. J. Ferri, J. M. Iñesta, A. Amin, \& P. Pudil (Eds.), Advances in pattern recognition (pp. 621-630). Springer.

16. Beigman, E., \& Klebanov, B. B. (2009). Learning with annotation noise. In Proceedings of the joint conference of the 47th annual meeting of the ACL and the 4th international joint conference on natural language processing of the AFNLP: Volume 1, ACL 'O9 (Vol. 1, pp. 280-287). Association for Computational Linguistics.

17. Bellman, R. (1957). Dynamic programming. Princeton University Press.

18. Ben-Akiva, M., \& Lerman, S. R. (1985). Discrete choice analysis: Theory and application to travel demand. The MIT Press.

19. Bierlaire, M., Chen, J., \& Newman, J. (2013). A probabilistic map matching method for smartphone GPS data. Transportation Research Part C: Emerging Technologies, 26, 78-98.

20. Blum, J. R., Greencorn, D. G., \& Cooperstock, J. R. (2013). Smartphone sensor reliability for augmented reality applications. In K. Zheng, M. Li, \& H. Jiang (Eds.), Mobile and ubiquitous systems: Computing, networking, and services (pp. 127-138). Springer.

21. Bohte, W., \& Maat, K. (2009). Deriving and validating trip purposes and travel modes for multi-day GPS-based travel surveys: A large-scale application in The Netherlands. Transportation Research Part C: Emerging Technologies, 17(3), 285-297.

22. Byon, Y. J., \& Liang, S. (2014). Real-time transportation mode detection using smartphones and artificial neural networks: Performance comparisons between smartphones and conventional global positioning system sensors. Journal of Intelligent Transportation Systems: Technology, Planning, and Operations, 18, 264-272.

23. Calastri, C., Dit Sourd, R. C., \& Hess, S. (2018). We want it all: Experiences from a survey seeking to capture social network structures, lifetime events and short-term travel and activity planning. Transportation, 47, 175-201.

24. Carpineti, C., Lomonaco, V., Bedogni, L., Felice, M. D., \& Bononi, L. (2018). Custom dual transportation mode detection by smartphone devices exploiting sensor diversity. In Proceedings of the 14th workshop on context and activity modeling and recognition (IEEE COMOREA 2018).

25. Chavarriaga, R., Sagha, H., Calatroni, A., Digumarti, S. T., Tröster, G., del Millán, J. R., \& Roggen, D. (2013). The opportunity challenge: A benchmark database for on-body sensor-based activity recognition. Pattern Recognition Letters, 34(15), 2033-2042. Smart Approaches for Human Action Recognition.

26. Chen, J., \& Bierlaire, M. (2015). Probabilistic multimodal map matching with rich smartphone data. Journal of Intelligent Transportation Systems: Technology, Planning, and Operations, 19(2), 134-148.

27. Christensen, L. (2013). The Role of Web Interviews as Part of a National Travel Survey. In J. Zmud, M. Lee-Gosselin, M. Munizaga, \& J. A. Carrasco (Eds.), Transport Survey Methods (pp. 115-154). Emerald Group Publishing Limited. https://doi.org/10.1108/9781781902882-006.
28. Christiansen, H. (Author), \& Warnecke, M-L. (Author). (2018). The Danish National Travel Survey - declaration of variables TU 2006-17, version 1. Dataset, DTU Management.

29. Cornacchia, M., Ozcan, K., Zheng, Y., \& Velipasalar, S. (2017). A survey on activity detection and classification using wearable sensors. IEEE Sensors Journal, 17(2), 7742959.

30. Cottrill, C., Pereira, F., Zhao, F., Dias, I., Lim, H., Ben-Akiva, M., \& Zegras, P. (2013). Future mobility survey. Transportation Research Record: Journal of the Transportation Research Board, 2354, 59-67.

31. Dabiri, S., \& Heaslip, K. (2018). Inferring transportation modes from GPS trajectories using a convolutional neural network. Transportation Research Part C: Emerging Technologies, 86(November 2017), 360-371.

32. Dabiri, S., Lu, C.-T., Heaslip, K., \& Reddy, C. K. (2019). Semi-supervised deep learning approach for transportation mode identification using GPS trajectory data. IEEE Transactions on Knowledge and Data Engineering, 32, 1010-1023.

33. Das, R. D., \& Winter, S. (2016). Automated urban travel interpretation: A bottom-up approach for trajectory segmentation. Sensors (Switzerland), 16(11), 1962.

34. Davidson, P., \& Piché, R. (2017). A survey of selected indoor positioning methods for smartphones. IEEE Communications Surveys Tutorials, 19(2), 1347-1370.

35. De Montjoye, Y. A., Hidalgo, C. A., Verleysen, M., \& Blondel, V. D. (2013). Unique in the crowd: The privacy bounds of human mobility. Scientific Reports, 3, 1-5.

36. Ectors, W., Reumers, S., Lee, W. D., Choi, K., Kochan, B., Janssens, D., Bellemans, T., \& Wets, G. (2017). Developing an optimised activity type annotation method based on classification accuracy and entropy indices. Transportmetrica A: Transport Science, 13(8), 742-766.

37. Ehsani, R., Buchanon, S., \& Salyani, M. (2009). GPS Accuracy for Tree Scouting and Other Horticultural Uses. EDIS, 2009(2). Retrieved from https://journals.flvc.org/edis/article/view/117815.

38. Ek, A., Alexandrou, C., Delisle Nyström, C., Direito, A., Eriksson, U., Hammar, U., Henriksson, P., Maddison, R., Trolle Lagerros, Y., \& Löf, M. (2018). The Smart City Active Mobile Phone Intervention (SCAMPI) study to promote physical activity through active transportation in healthy adults: A study protocol for a randomised controlled trial. BMC Public Health, 18, 1-11.

39. Faouzi, N. E. E., Leung, H., \& Kurian, A. (2011). Data fusion in intelligent transportation systems: Progress and challenges-A survey. Information Fusion, 12, 4-10.

40. Feng, T., \& Timmermans, H. J. (2015). Detecting activity type from GPS traces using spatial and temporal information. European Journal of Transport and Infrastructure Research, 15(4), 662-674.

41. Gadziński, J. (2018). Perspectives of the use of smartphones in travel behaviour studies: Findings from a literature review and a pilot study. Transportation Research Part C: Emerging Technologies, 88(July 2017), 74-86.

42. Garg, N. (2018). Mining bus stops from raw GPS data of bus trajectories. In 10th International conference on communication systems \& networks (COMSNETS), Bengaluru, India (pp. 583-588). IEEE.

43. Geurs, K. T., Thomas, T., Bijlsma, M., \& Douhou, S. (2015). Automatic trip and mode detection with move smarter: first results from the dutch mobile mobility panel. Transport Res Proc., https://doi.org/10.1016/j. trpro.2015.12.022

44. Gong, L., Morikawa, T., Yamamoto, T., \& Sato, H. (2014). Deriving personal trip data from GPS data: A literature review on the existing methodologies. Procedia-Social and Behavioral Sciences, 138, 557-565.

45. Greaves, S., Ellison, A., Ellison, R., Rance, D., Standen, C., Rissel, C., \& Crane, M. (2015). A web-based diary and companion smartphone app for travel/activity surveys. Transportation Research Procedia, 11, 297-310.

46. Guidotti, R., Trasarti, R., \& Nanni, M. (2015). TOSCA: Two-steps clustering algorithm for personal locations detection. In GIS: Proceedings of the ACM international symposium on advances in geographic information systems.

47. Hall, M., Frank, E., Holmes, G., Pfahringer, B., Reutemann, P., \& Witten, I. H. (2009). The WEKA data mining software: An update. S/GKDD Explorations Newsletter, 11(1), 10-18.

48. Hariharan, R., \& Toyama, K. (2004). Project lachesis: Parsing and modeling location histories. In M. J. Egenhofer, C. Freksa, \& H. J. Miller (Eds.), Geographic information science (pp. 106-124). Springer. 
49. Hoseini-Tabatabaei, S. A., Gluhak, A., \& Tafazolli, R. (2013). A survey on smartphone-based systems for opportunistic user context recognition. ACM Computing Surveys, 45(3), 1-51.

50. Houston, D., Luong, T. T., \& Boarnet, M. G. (2014). Tracking daily travel; Assessing discrepancies between GPS-derived and self-reported travel patterns. Transportation Research Part C: Emerging Technologies, 48, 97-108.

51. Huang, J., Qiao, S., Yu, H., Qie, J., \& Liu, C. (2014). Parallel map matching on massive vehicle GPS data using MapReduce. In Proceedings-2013 IEEE international conference on high performance computing and communications, HPCC 2013 and 2013 IEEE international conference on embedded and ubiquitous computing, EUC 2013 (pp. 1498-1503).

52. Hunter, T., Abbeel, P., \& Bayen, A. (2014). The path inference filter: Modelbased low-latency map matching of probe vehicle data. IEEE Transactions on Intelligent Transportation Systems, 15(2), 507-529.

53. Iqbal, M. S., Choudhury, C. F., Wang, P., \& González, M. C. (2014). Development of origin-destination matrices using mobile phone call data. Transportation Research Part C: Emerging Technologies, 40, 63-74.

54. Jagadeesh, G. R., \& Srikanthan, T. (2017). Online map-matching of noisy and sparse location data with hidden Markov and route choice models. IEEE Transactions on Intelligent Transportation Systems, 18, 2423-2434.

55. Jahangiri, A., \& Rakha, H. A. (2015). Applying machine learning techniques to transportation mode recognition using mobile phone sensor data. IEEE Transactions on Intelligent Transportation Systems, 16(5), 2406-2417.

56. Jeon, K. E., She, J., Soonsawad, P., \& Ng, P. C. (2018). BLE beacons for internet of things applications: Survey, challenges, and opportunities. IEEE Internet of Things Journal, 5(2), 811-828.

57. Jiang, X., de Souza, E. N., Pesaranghader, A., Hu, B., Silver, D. L., \& Matwin, S. (2017). TrajectoryNet: An embedded GPS trajectory representation for point-based classification using recurrent neural networks. Source code published on Github@https://github.com/wuhaotju/TrajectoryNet. Retrieved November 1, 2019, from web.

58. Kanarachos, S., Christopoulos, S. R. G., \& Chroneos, A. (2018). Smartphones as an integrated platform for monitoring driver behaviour: The role of sensor fusion and connectivity. Transportation Research Part C: Emerging Technologies, 95(March), 867-882.

59. Karlaftis, M. G., \& Vlahogianni, E. I. (2011). Statistical methods versus neural networks in transportation research: Differences, similarities and some insights. Transportation Research Part C: Emerging Technologies, 19(3), 387-399.

60. Kim, Y., Pereira, F. C., Zegras, P. C., \& Ben-akiva, M. (2018). Activity recognition for a smartphone and web-based human mobility sensing system. IEEE Intelligent Systems, 33(August), 5-23.

61. Kiukkonen, N., Blom, J., Dousse, O., Gatica-Perez, D., \& Laurila, J. (2010). Towards rich mobile phone datasets: Lausanne data collection campaign. Proc. ICPS, Berlin, 68, 7

62. Koushik, A. N., Manoj, M., \& Nezamuddin, N. (2020). Machine learning applications in activity-travel behaviour research: A review. Transport Reviews, 40, 1-24.

63. Kubicka, M., Cela, A., Moulin, P., Mounier, H., \& Niculescu, S. I. (2016). Dataset for testing and training map-matching methods [Data set]. 2015 IEEE Intelligent Vehicles Symposium (IV 2015), Seoul, South Korea. Zenodo. https://doi.org/10.5281/zenodo.57731.

64. Kubicka, M., Cela, A., Mounier, H., \& Niculescu, S. I. (2018). Comparative study and application-oriented classification of vehicular map-matching methods. IEEE Intelligent Transportation Systems Magazine, 10(2), 150-166.

65. Laurila, J. K., Gatica-Perez, D., Aad, I., Blom, J., Bornet, O., Do, T. M. T., Dousse, O., Eberle, J., \& Miettinen, M. (2013). From big smartphone data to worldwide research: The mobile data challenge. Pervasive and Mobile Computing, 9(6), 752-771.

66. Li, C., Zegras, P. C., Zhao, F., Qin, Z., Shahid, A., Ben-Akiva, M., Pereira, F., \& Zhao, J. (2017). Enabling bus transit service quality co-monitoring through smartphone-based platform. Transportation Research Record: Journal of the Transportation Research Board, 2649(1), 42-51.

67. Li, H., \&Wu, G. (2014). Map matching for taxi GPS data with extreme learning machine (Vol. 8933). Springer.

68. Li, L., Quddus, M., \& Zhao, L. (2013). High accuracy tightly-coupled integrity monitoring algorithm for map-matching. Transportation Research Part C: Emerging Technologies, 36, 13-26.
69. Li, X., Zhang, X., Chen, K., \& Feng, S. (2014). Measurement and analysis of energy consumption on android smartphones. In 2014 4th IEEE International conference on information science and technology (pp. 242-245).

70. Liao, L., Fox, D., \& Kautz, H. (2007). Extracting places and activities from GPS traces using hierarchical conditional random fields. International Journal of Robotics Research, 26, 119-134.

71. Lou, Y., Zhang, C., Zheng, Y., Xie, X., Wang, W., \& Huang, Y. (2009). Mapmatching for low-sampling-rate GPS trajectories. In Proceedings of the 17th ACM SIGSPATIAL international conference on advances in geographic information systems-GIS '09, (c) (p. 352).

72. Mäenpää, H., Lobov, A., \& Martinez Lastra, J. L. (2017). Travel mode estimation for multi-modal journey planner. Transportation Research Part C: Emerging Technologies, 82, 273-289.

73. Teng, C. M. (2001, May). A Comparison of Noise Handling Techniques. In Proceedings of the Fourteenth International Florida Artificial Intelligence Research Society Conference (pp. 269-273).

74. Manwani, N., \& Sastry, P. S. (2013). Noise tolerance under risk minimization. IEEE Transactions on Cybernetics, 43(3), 1146-1151.

75. Martin, B. D., Addona, V., Wolfson, J., Adomavicius, G., \& Fan, Y. (2017). Methods for real-time prediction of the mode of travel using smartphone-based GPS and accelerometer data. Sensors (Switzerland), 17(9), 2058.

76. Montini, L., Rieser-Schüssler, N., Horni, A., \& Axhausen, K. (2014). Trip purpose identification from GPS tracks. Transportation Research Record: Journal of the Transportation Research Board, 2405, 16-23.

77. Nettleton, D. F., Orriols-Puig, A., \& Fornells, A. (2010). A study of the effect of different types of noise on the precision of supervised learning techniques. Artificial Intelligence Review, 33(4), 275-306.

78. Newson, P., \& Krumm, J. (2009). Hidden Markov map matching through noise and sparseness. In Proceedings of the 17th ACM SIGSPATIAL international conference on advances in geographic information systems-GIS '09 (pp. 336-343).

79. Nicholls, L., II., \& Groves, R. M. (1986). The status of computer-assisted telephone interviewing: Part I-Introduction and impact on cost and timeliness of survey data. Journal of Official Statistics, 2(2), 93.

80. Nitsche, P., Widhalm, P., Breuss, S., Brändle, N., \& Maurer, P. (2014). Supporting large-scale travel surveys with smartphones-A practical approach. Transportation Research Part C: Emerging Technologies, 43, 212-221

81. Nurmi, P., \& Koolwaaij, J. (2006). Identifying meaningful locations. In 2006 3rd Annual international conference on mobile and ubiquitous systems: Networking and services, MobiQuitous.

82. Oshin, T. O., Poslad, S., \& Ma, A. (2012). Improving the energy-efficiency of GPS based location sensing smartphone applications. In Proceedings of the 11th IEEE international conference on trust, security and privacy in computing and communications, TrustCom-2012-11th IEEE international conference on ubiquitous computing and communications, IUCC-2012 (pp. 1698-1705).

83. Patterson, Z., \& Fitzsimmons, K. (2016). Datamobile: Smartphone travel survey experiment. Transportation Research Record, 2594, 35-53.

84. Patterson, Z., Fitzsimmons, K., Jackson, S., \& Mukai, T. (2019). Itinerum: The open smartphone travel survey platform. SoftwareX, 10, 100230.

85. Perrucci, G. P., Fitzek, F. H. P., \& Widmer, J. (2011). Survey on energy consumption entities on the smartphone platform. In 2011 IEEE 73rd Vehicular technology conference (VTC Spring) (pp. 1-6).

86. Prelipcean, A. C., Gidofalvi, G., \& Susilo, Y. O. (2016). Measures of transport mode segmentation of trajectories. International Journal of Geographical Information Science, 30(9), 1763-1784.

87. Prelipcean, A. C., Gidófalvi, G., \& Susilo, Y. O. (2018). MEILI: A travel diary collection, annotation and automation system. Computers, Environment and Urban Systems, 70, 24-34.

88. Primault, V., Boutet, A., Mokhtar, S. B., \& Brunie, L. (2019). The long road to computational location privacy: A survey. IEEE Communications Surveys and Tutorials, 21(3), 8482357, 2772-2793.

89. Quddus, M. A., Noland, R. B., \& Ochieng, W. Y. (2006). A high accuracy fuzzy logic based map matching algorithm for road transport. Journal of Intelligent Transportation Systems: Technology, Planning, and Operations, 10(3), 103-115.

90. Rasmussen, T. K., Ingvardson, J. B., Halldórsdóttir, K., \& Nielsen, O. A. (2015). Improved methods to deduct trip legs and mode from travel surveys using wearable GPS devices: A case study from the Greater 
Copenhagen area. Computers, Environment and Urban Systems, 54, 301-313.

91. Renso, C., Baglioni, M., de Macedo, J. A. F., Trasarti, R., \& Wachowicz, M. (2013). How you move reveals who you are: Understanding human behavior by analyzing trajectory data. Knowledge and Information Systems, 37(2), 331-362.

92. Rolnick, D., Veit, A., Belongie, S., \& Shavit, N. (2018). Deep learning is robust to massive label noise. Retrieved November 14, 2019, from the arXiv database.

93. Rosvall, M., Axelsson, D., \& Bergstrom, C. T. (2009). The map equation. European Physical Journal Special Topics, 178(1), 13-23.

94. Schuessler, N., \& Axhausen, K. W. (2009). Processing raw data from global positioning systems without additional information. Transportation Research Record, 2105(1), 28-36.

95. Seidl, D. E., Jankowski, P., \& Tsou, M. H. (2016). Privacy and spatial pattern preservation in masked GPS trajectory data. International Journal of Geographical Information Science, 30(4), 785-800.

96. Semanjski, I., Gautama, S., Ahas, R., \& Witlox, F. (2017). Spatial context mining approach for transport mode recognition from mobile sensed big data. Computers, Environment and Urban Systems, 66, 38-52.

97. Shankari, K., Fürst, J., Fadel Argerich, M., Avramidis, E., \& Zhang, J. (2020). MobilityNet:Towards a Public Dataset for Multi-modal Mobility Research. ICLR 2020 Workshop on Tackling Climate Change with Machine Learning. https://www.climatechange.ai/papers/iclr2020/15.html.

98. Shen, L., \& Stopher, P. R. (2014). Review of GPS travel survey and GPS data-processing methods, Transport Reviews, 34:3, 316-334. https://doi. org/10.1080/01441647.2014.903530.

99. Sicotte, G., Morency, C., \& Farooq, B. (2017). Comparison between trip and trip chain models: Evidence from Montreal commuter train corridor (No. CIRRELT-2017-35). CIRRELT, Centre interuniversitaire de recherche sur les réseaux d'entreprise, la logistique et le transport = Interuniversity Research Centre on Enterprise Networks, Logistics and Transportation.

100. Silver, D. L., Yang, Q., \& Li, L. (2013). Lifelong machine learning systems: Beyond learning algorithms. In 2013 AAAl spring symposium series. Citeseer.

101. Stopher, P. R., \& Greaves, S. P. (2007). Household travel surveys: Where are we going? Transportation Research Part A: Policy and Practice, 41(5), 367-381.

102. Stopher, P. R., Shen, L., Liu, W., \& Ahmed, A. (2015). The challenge of obtaining ground truth for GPS processing. Transportation Research Procedia, 11, 206-217. Transport Survey Methods: Embracing Behavioural and Technological Changes Selected contributions from the 10th International Conference on Transport Survey Methods 16-21 November 2014, Leura, Australia.

103. Thierry, B., Chaix, B., \& Kestens, Y. (2013). Detecting activity locations from raw GPS data: A novel kernel-based algorithm. International Journal of Health Geographics, 12, 1-10.

104. Thomas, T., Geurs, K. T., Koolwaaij, J., \& Bijlsma, M. (2018). Automatic trip detection with the dutch mobile mobility panel: Towards reliable multiple-week trip registration for large samples. Journal of Urban Technology, 25, 1-19.

105. Tietbohl, A., Bogorny, V., Kuijpers, B., \& Alvares, L. O. (2008). A clusteringbased approach for discovering interesting places in trajectories. In Proceedings of the ACM symposium on applied computing.

106. Torre, F., Pitchford, D., Brown, P., \& Terveen, L. (2012). Matching GPS traces to (possibly) incomplete map data. In Proceedings of the 20th international conference on advances in geographic information systems-SIGSPATIAL'12 (p. 546).

107. Van Dijk, J. (2018). Identifying activity-travel points from GPS-data with multiple moving windows. Computers, Environment and Urban Systems, 70(September 2017), 84-101.

108. Velasco-Gallego, C., \& Lazakis, I. (2020). Real-time data-driven missing data imputation for short-term sensor data of marine systems. A comparative study. Ocean Engineering, 218, 108261.

109. von Watzdorf, S., \& Michahelles, F. (2010). Accuracy of positioning data on smartphones. In Proceedings of the 3rd international workshop on location and the web, LocWeb'10, New York. Association for Computing Machinery.
110. Vuk, G., Bowman, J. L., Daly, A., \& Hess, S. (2016). Impact of family in-home quality time on person travel demand. Transportation, 43(4), 705-724.

111. Wang, D., Zhang, J., Cao, W., Li, J., \& Zheng, Y. (2018). When will you arrive? Estimating travel time based on deep neural networks. In IJCAI.

112. Wang, L., Gjoreski, H., Ciliberto, M., Mekki, S., Valentin, S., \& Roggen, D. (2019). Enabling reproducible research in sensor-based transportation mode recognition with the Sussex-Huawei dataset. IEEE Access, 7, 10870-10891.

113. Wang, L., Jiao, L., Li, J., Gedeon, J., \& Mühlhäuser, M. (2019). Moera: Mobility-agnostic online resource allocation for edge computing. IEEE Transactions on Mobile Computing, 18(8), 1843-1856.

114. Wee, B. V., \& Banister, D. (2016). How to write a literature review paper? Transport Reviews, 36(2), 278-288.

115. Wei, H., Wang, Y., Forman, G., \& Zhu, Y. (2013). Map matching: Comparison of approaches using sparse and noisy data. In Proceedings of the 21st ACM SIGSPATIAL international conference on advances in geographic information systems, SIGSPATIAL'13, New York (pp. 444-447). Association for Computing Machinery.

116. Wu, H., Chen, Z., Sun, W., Zheng, B., \& Wang, W. (2017). Modeling trajectories with recurrent neural networks. In IJCAI International joint conference on artificial intelligence (pp. 3083-3090).

117. Xiang, L., Gao, M., \& Wu, T. (2016). Extracting stops from noisy trajectories: A sequence oriented clustering approach. ISPRS International Journal of Geo-Information, 5, 29.

118. Xiao, G., Cheng, Q., \& Zhang, C. (2019). Detecting travel modes from smartphone-based travel surveys with continuous hidden Markov models. International Journal of Distributed Sensor Networks, 15, 1550147719844156

119. Xiao, G., Juan, Z., \& Zhang, C. (2015). Travel mode detection based on GPS track data and Bayesian networks. Computers, Environment and Urban Systems, 54, 14-22.

120. Xiao, G., Juan, Z., \& Zhang, C. (2016). Detecting trip purposes from smartphone-based travel surveys with artificial neural networks and particle swarm optimization. Transportation Research Part C: Emerging Technologies, 71, 447-463.

121. Xiao, L., Li, Y., Han, G., Dai, H., \& Poor, H. V. (2018). A secure mobile crowdsensing game with deep reinforcement learning. IEEE Transactions on Information Forensics and Security, 13(1), 35-47.

122. Yazdizadeh, A., Patterson, Z., \& Farooq, B. (2019). An automated approach from GPS traces to complete trip information. International Journal of Transportation Science and Technology, 8, 82-100.

123. Yazdizadeh, A., Patterson, Z., \& Farooq, B. (2019). Ensemble convolutional neural networks for mode inference in smartphone travel survey. IEEE Transactions on Intelligent Transportation Systems, 21, 2232-2239.

124. Kalatian, A., \& Farooq, B. (2020). A semi-supervised deep residual network for mode detection in Wi-Fi signals. Journal of Big Data Analytics in Transportation, 2(2), 167-180.

125. Yurur, O., Liu, C. H., Sheng, Z., Leung, V. C. M., Moreno, W., \& Leung, K. K. (2016). Context-awareness for mobile sensing: A survey and future directions. IEEE Communications Surveys and Tutorials, 18(1), 68-93.

126. Zhao, F., Ghorpade, A., Pereira, F. C., Zegras, C., \& Ben-Akiva, M. (2015a). Stop detection in smartphone-based travel surveys. Transportation Research Procedia, 11(2010), 218-226.

127. Zhao, F., Pereira, F. C., Ball, R., Kim, Y., Han, Y., Zegras, C., \& Ben-Akiva, M. (2015b). Exploratory analysis of a smartphone-based travel survey in Singapore. Transportation Research Record, 2494(1), 45-56.

128. Zheng, Y. (2015). Trajectory data mining: An overview. ACM Transactions on Intelligent Systems and Technology (TIST), 6(3), 1-41.

129. Zheng, Y., \& Fu, H. (2011). Geolife GPS trajectory dataset-User guide. Technical Report November 31. Online. Retrieved July 19, 2008.

130. Zheng, Y., Zhang, L., Xie, X., \& Ma, W.-Y. (2009). Mining interesting locations and travel sequences from GPS trajectories. In Proceedings of the 18th international conference on world wide web-WWW'09.

131. Zhou, R., Li, M., Wang, H., Song, X., Xie, W., \& Lu, Z. (2017). An enhanced transportation mode detection method based on GPS data. Communications in Computer and Information Science, 727, 605-620.

132. Zhou, X., Yu, W., \& Sullivan, W. C. (2016). Making pervasive sensing possible: Effective travel mode sensing based on smartphones. Computers, Environment and Urban Systems, 58, 52-59. 
133. Zhu, Q., Zhu, M., Li, M., Fu, M., Huang, Z., Gan, Q., \& Zhou, Z. (2016). Identifying transportation modes from raw GPS data. In Communications in computer and information science.

134. Zhu, X., Li, J., Liu, Z., Wang, S., \& Yang, F. (2016). Learning transportation annotated mobility profiles from GPS data for context-aware mobile services. In Proceedings - 2016 IEEE international conference on services computing, SCC 2016 (pp. 475-482)

135. Zmud, J., Lee-Gosselin, M., Carrasco, J. A., \& Munizaga, M. A. (2013) Transport survey methods: Best practice for decision making. Emerald Group Publishing.

\section{Publisher's Note}

Springer Nature remains neutral with regard to jurisdictional claims in published maps and institutional affiliations.

\section{Submit your manuscript to a SpringerOpen ${ }^{\odot}$ journal and benefit from:}

- Convenient online submission

- Rigorous peer review

- Open access: articles freely available online

- High visibility within the field

- Retaining the copyright to your article

Submit your next manuscript at $\boldsymbol{\nabla}$ springeropen.com 